\title{
Synthesis of a New (1R)-(-)-Myrtenal Derived Dioxadithiadodecacycle and its Use as Efficient Chiral Auxiliary
}

M. Elena Vargas-Díaz, Pedro Joseph-Nathan, Joaquín Tamariz, and L. Gerardo Zepeda

Experimental

\section{General}

Melting points were determined on an Electrothermal capillary melting point apparatus and are uncorrected. Optical rotations were measured at $589 \mathrm{~nm}$ using a $1 \mathrm{dm}$ cell on a JASCO DIP-370 polarimeter. Infrared spectra were recorded on a Perkin-Elmer Spectrum 2000 spectrophotometer in $\mathrm{KBr}$ pellets or film as specified. ${ }^{1} \mathrm{H}$ and ${ }^{13} \mathrm{C}$ NMR spectra were obtained on a Varian Mercury spectrometer at 300 and $75.4 \mathrm{MHz}$, as specified, using $\mathrm{CDCl}_{3}$ as solvent and TMS as internal standard. The low-resolution mass spectra (LRMS) were recorded on a HP 5971A or a Varian Saturn 2000 GC/Selective Mass Detector, using either electronic impact (EI, $70 \mathrm{eV}$ ) or chemical ionization $(\mathrm{Cl})$, as specified. The highresolution electron impact mass spectra (HREIMS) were recorded at the UCR Mass Spectrometry Facility, University of California, Riverside, CA. Elemental analyses were determined by A-M-W laboratories, Phoenix, AZ. Thin layer chromatograms were done on precoated TLC sheets of silica gel 60 F254 (E. Merck). Flash chromatography was carried out using silica gel (Merck 230-400 mesh). THF and benzene were distilled from $\mathrm{Na}$ immediately prior to use, and all other reagents were used without further purification.

\section{2,2-Bis-[(1S,1'S,2R,2'R,3S,3'S)-2-hydroxymethyl-6,6-dimethylbicyclo[3.1.1]hept-3- ylsulfanyl]-1-phenylethanone (11a)}

A vigorously stirred solution of $3 \mathrm{~g}(16.1 \mathrm{mmol})$ of hydroxythiol 10, $1.67 \mathrm{~g}(8.06 \mathrm{mmol})$ of 2,2-diethoxyacetophenone and $1 \mathrm{~mL}$ of $\mathrm{BF}_{3} . \mathrm{OEt}_{2}$ in $10 \mathrm{~mL}$ of $\mathrm{CHCl}_{3}$ was allowed to react at room temperature for $3 \mathrm{~h}$. After addition of $300 \mathrm{~mL}$ of $\mathrm{CH}_{2} \mathrm{Cl}_{2}$, the reaction mixture was washed with a saturated solution of $\mathrm{NaHCO}_{3}(4 \times 50 \mathrm{~mL})$, the organic layer was dried with 
anhydrous $\mathrm{Na}_{2} \mathrm{SO}_{4}$ and evaporated to dryness. The residue was flash chromatographed using a mixture of hexane/EtOAc (4:1) as the eluent, obtaining $3.1 \mathrm{~g}(78 \%)$ of bis-sulfide 11a as a white solid, mp 59-60 ${ }^{\circ} \mathrm{C} .[\alpha]^{25}{ }_{\mathrm{D}}=+109.6\left(\mathrm{c}=1.0, \mathrm{CHCl}_{3}\right) .{ }^{1} \mathrm{H} \mathrm{NMR}(300 \mathrm{MHz}$, $\left.\mathrm{CDCl}_{3}\right): \delta 8.04(\mathrm{~d}, 2 \mathrm{H}, J=7.2 \mathrm{~Hz}, \mathrm{H}-o), 7.57(\mathrm{t}, 2 \mathrm{H}, J=7.2 \mathrm{~Hz}, \mathrm{H}-p), 7.56(\mathrm{t}, 2 \mathrm{H}, J=7.8$ Hz, H-m), 5.60 (s, 1H, H-11), 3.82-3.67 (m, 2H, H-10a,b and H-10'a,b), 3.40-3.28 (m, 1H, H-3, H-3'), 2.58-2.30 (m, 6H, H-4eq, H-4'eq, H-2, H-2', H-7eq, H-7'eq), 2.19-1.80 (m, 6H, H-5, H- 5', H-4ax, H-4'ax, H-1, H-1'), 1.21 (s, 3H, Me-9), 1.17 (s, 3H, Me-9'), 1.12 (d, $1 \mathrm{H}, J=10.1 \mathrm{~Hz}, \mathrm{H}-7 \mathrm{ax}), 1.03$ (d, 1H, $J=9.9 \mathrm{~Hz}, \mathrm{H}-7$ 'ax), 0.97 (s, 3H, Me-8), 0.93 (s, 3H, Me-8'). ${ }^{13} \mathrm{C}$ NMR (75.4 MHz, $\left.\mathrm{CDCl}_{3}\right): \delta 194.2(\mathrm{CO}), 134.5(\mathrm{C}-i), 133.6(\mathrm{C}-o), 129.1(\mathrm{C}-$ m), 128.6 (C-p), 66.4 (C-10,10’), 54.2 (C-11), 53.0 (C-2), 52.2 (C-2'), 43.7 (C-1), 43.6 (C1'), 41.8 (C-5), 41.6 (C-5'), 38.2 (C-6), 38.0 (C-4), 37.9 (C-4'), 37.8 (C-6'), 36.5 (C-3), 36.3 (C-3’), 32.6 (C-7), 32.4 (C-7'), 27.3 (C-9), 27.2 (C-9’), 23.7 (C-8,8') . IR (KBr): $v_{\max }$ $3425,2922,1449,1384,1045,687 \mathrm{~cm}^{-1}$. EA calcd. for $\mathrm{C}_{28} \mathrm{H}_{40} \mathrm{O}_{3} \mathrm{~S}_{2}$ : C, 68.81; H, 8.25; S, 13.12. Found: C, 68.70; H, 8.12; S, 13.13 .

\section{2,2-Bis-[(1S,1'S,2R,2' $R, 3 S, 3$ 'S)-2-(tert-butyl-dimethylsilanyloxymethyl)-6,6-dimethyl- bicyclo[3.1.1]hept-3-ylsulfanyl]-1-phenylethanone (11b)}

To a solution of $100 \mathrm{mg}(0.20 \mathrm{mmol})$ of $11 \mathrm{a}$ in $3 \mathrm{~mL}$ of $\mathrm{CH}_{2} \mathrm{Cl}_{2}$ were added $0.02 \mathrm{~mL}$ of triethylamine, $154 \mathrm{mg}(1.02 \mathrm{mmol})$ of $\mathrm{TBSCl}$ and $25 \mathrm{mg}(0.20 \mathrm{mmol})$ of 4dimethylaminopyridine. The resulting mixture was stirred during $4 \mathrm{~h}$. The organic layer was washed with a saturated solution of ammonium chloride, dried over anhydrous $\mathrm{Na}_{2} \mathrm{SO}_{4}$

and concentrated to dryness, giving $136 \mathrm{mg}(95 \%)$ of $\mathbf{1 1 b}$ as a colorless oil. $[\alpha]^{25}=+89.6$ $\left(\mathrm{c}=0.27, \mathrm{CHCl}_{3}\right) .{ }^{1} \mathrm{H} \mathrm{NMR}\left(300 \mathrm{MHz}, \mathrm{CDCl}_{3}\right): \delta 8.05(\mathrm{~d}, 2 \mathrm{H}, J=7.4 \mathrm{~Hz}, \mathrm{H}-o), 7.48(\mathrm{t}$, $2 \mathrm{H}, J=7.4 \mathrm{~Hz}, \mathrm{H}-m), 7.45$ (t, 1H, $J=7.4 \mathrm{~Hz}, \mathrm{H}-p), 5.31$ (s, 1H, H-11), 3.62-3.45 (m, 4H, H-10a,b and H-10'a,b), 3.10-3.00 (m, 2H, H-3, H-3'), 2.66-1.82 (m, 12H, H-4eq, H-4'eq, H-7eq, H-7'eq, H-4ax, H-4'ax, H-2, H-2', H-1, H-1', H-5, H-5'), 1.21 (s, 3H, Me-9), 1.17 (s, 3H, Me-9'), 1.00 (s, 3H, Me-8), 0.93 (s, 3H, Me-8'), 0.89-0.84 (m, 11H, H-7ax, 7'ax, $\left.\mathrm{C}\left(\mathrm{CH}_{3}\right)_{3}\right), 0.10\left(\mathrm{~s}, 6 \mathrm{H}, \mathrm{Si}(\mathrm{Me})_{2}\right) .{ }^{13} \mathrm{C} \mathrm{NMR}\left(75.4 \mathrm{MHz}, \mathrm{CDCl}_{3}\right): \delta 193.6(\mathrm{CO}), 134.6(\mathrm{C}-i)$, $133.3(\mathrm{C}-o), 129.2$ (C-m), 128.7 (C-p), 65.4 (C-10), 65.1 (C-10’), 53.4 (C-11), 52.1 (C-2), 51.5 (C-2'), 42.1 (C-1), 42.0 (C-1'), 41.9 (C-5), 41.7 (C-5'), 38.5 (C-6), 38.4 (C-6'), 38.2 
(C-4), 37.7 (C-4'), 35.5 (C-3), 35.2 (C-3’), 33.1 (C-7), 32.8 (C-7’), 27.6 (C-9), 27.6 (C-9'), $25.9\left(\mathrm{C}\left(\mathrm{CH}_{3}\right)_{3}\right), 23.4(\mathrm{C}-8), 18.3\left(\underline{\mathrm{C}}-\left(\mathrm{CH}_{3}\right)_{3}\right),-5.2\left(\mathrm{Si}-\left(\mathrm{CH}_{3}\right)_{2}\right)$. IR (Film): $v_{\max } 3425,2922$, $1449,1384,1045,687 \mathrm{~cm}^{-1}$.

$(1 S, 2 R, 8 R, 9 S, 13 S, 17 S)-4,6-D i o x a-14,16-d i t h i a-15-b e n z o y l-10,10,20,20-$ tetramethylpentacyclo-[17.1.1.1 $\left.{ }^{9,11} \cdot 0^{2,17} \cdot 0^{8,13}\right]$-docosane (9)

A vigorously stirred solution of $500 \mathrm{mg}(1.02 \mathrm{mmol})$ of bis-sulfide 11a, $116 \mathrm{mg}(1.52$ mmol) of dimethoxymetane and $50 \mathrm{mg}$ of $p \mathrm{TsOH}$ in $8 \mathrm{~mL}$ of benzene was refluxed for $3 \mathrm{~h}$.

The reaction mixture was poured into a cold saturated solution of $\mathrm{NaHCO}_{3}$, extracted with $\mathrm{CH}_{2} \mathrm{Cl}_{2}$, washed with a saturated solution of $\mathrm{NaHCO}_{3}(2 \times 50 \mathrm{~mL})$, dried over anhydrous $\mathrm{Na}_{2} \mathrm{SO}_{4}$ and concentrated to dryness. The residue was flash chromatographed using a mixture of hexane/EtOAc (4:1) as the eluent, affording $460 \mathrm{mg}(91 \%)$ of dodecaheterocycle 9 as a white solid $\left(\mathrm{mp} 173-175{ }^{\circ} \mathrm{C}\right) .[\alpha]^{25} \mathrm{D}=+79.6\left(\mathrm{c}=2.50, \mathrm{CHCl}_{3}\right) .{ }^{1} \mathrm{H} \mathrm{NMR}(300 \mathrm{MHz}$, $\left.\mathrm{CDCl}_{3}\right): \delta 8.03(\mathrm{~d}, 2 \mathrm{H}, J=7.8 \mathrm{~Hz}, \mathrm{H}-o), 7.58(\mathrm{t}, 1 \mathrm{H}, J=7.8 \mathrm{~Hz}, \mathrm{H}-p), 7.47(\mathrm{t}, 2 \mathrm{H}, J=7.8$ Hz, H-m), 5.63 (s, 1H, H-15), 4.62 (d, 1H, $J=6.6 \mathrm{~Hz}, \mathrm{H}-5 \mathrm{a}), 4.59$ (d, 1H, $J=6.6 \mathrm{~Hz}, \mathrm{H}-$ 5b), 3.98 (dd, 1H, $J=9.9,3.3 \mathrm{~Hz}, \mathrm{H}-3 \mathrm{a}$ ), 3.85-3.69 (m, 3H, H-7a, H-17, H-13), 3.67-3.58 (m, 2H, H-3b, H-7b), 2.63 (m, 1H, H-12eq), 2.51-2.33 (m, 3H, H-21eq, H-22eq, H-18eq), 2.22-2.19 (m, 1H, H-2), 2.14-1.99 (m, 5H, H-8, H-1, H-9, H-11, H-12ax), 1.82 (m, 1H, H19), 1.69 (ddd, 1H, $J=11.5,6.0,2.7 \mathrm{~Hz}, \mathrm{H}-18 \mathrm{ax}$ ), 1.24 (s, 3H, Me-24), 1.17 (s, 3H, Me26), 1.13 (d, 1H, J = 9.9 Hz, H-21ax), 1.03 (s, 3H, Me-23), 1.01 (s, 3H, Me-25), 0.91 (d, $1 \mathrm{H}, J=9.9 \mathrm{~Hz}, \mathrm{H}-22 \mathrm{ax}) .{ }^{13} \mathrm{C}$ NMR $\left(75.4 \mathrm{MHz}, \mathrm{CDCl}_{3}\right.$ ): $\delta 194.3$ (CO), 134.9 (C-i), 133.2 (C-p), 128.9 (C-m), 126.6 (C-o), 93.8 (C-5), 71.1 (C-3), 69.9 (C-5), 54.2 (C-15), 50.9 (C-8), 49.8 (C-2), 47.0 (C-11), 46.9 (C-9), 42.0 (C-19), 42.0 (C-1), 38.6 (C-20), 38.3 (C-18), 36.9 (C-12), 35.6 (C-17), 34.8 (C-24), 34.6 (C-26), 34.5 (C-13), 27.8 (C-21), 27.7(C-23), 24.4 (C-22), 24.3 (C-25). IR (KBr): $v_{\max } 2918,1448,1048,700 \mathrm{~cm}^{-1}$. LRMS (EI) $\mathrm{m} / \mathrm{z}: 500(\mathrm{M})^{+}$, 395 (67), 197 (36), 135 (100.0), 93 (61). HRFABMS calcd. For $\mathrm{C}_{29} \mathrm{H}_{40} \mathrm{~S}_{2} \mathrm{O}_{3}: 500.2418$. Found: 500.2431.

General procedure for the addition of Grignard reagents to dodecaheterocycle 9 
To a solution of dodecaheterocycle 9 (1 equiv.) in anhydrous THF was added the Grignard reagent (1.5-4.0 equiv.) at $-78^{\circ} \mathrm{C}$ under a nitrogen atmosphere. After stirring for $2 \mathrm{~h}$ at the same temperature, the reaction mixture was allowed to warm up to room temperature and further stirred for $2 \mathrm{~h}$. The reaction mixture was quenched with $100 \mathrm{~mL}$ of a saturated solution of ammonium chloride, the THF was eliminated by evaporation and the remaining emulsion was extracted with ethyl ether. The organic layer was washed with a saturated solution of ammonium chloride, dried over anhydrous $\mathrm{Na}_{2} \mathrm{SO}_{4}$ and concentrated to dryness, giving the corresponding pure diastereoisomer $\mathbf{1 2}$ as a colorless oil or white solid, as specified.

\section{$(1 S, 2 R, 8 R, 9 S, 13 S, 17 S)-4,6-D i o x a-14,16-d i t h i a-15-[(R)-1-p h e n y l e t h-1-y l-1-o l]-$ $10,10,20,20$-tetramethylpentacyclo-[17.1.1.1 $\left.{ }^{9,11} \cdot 0^{2,17} \cdot 0^{8,13}\right]$-docosane (12a)}

To a solution of $200 \mathrm{mg}(0.40 \mathrm{mmol})$ of dodecaheterocycle 9 in anhydrous THF were added $0.54 \mathrm{~mL}(1.63 \mathrm{mmol})$ of $\mathrm{MeMgBr} 3 \mathrm{M}$ at $-78{ }^{\circ} \mathrm{C}$ in an $\mathrm{N}_{2}$ atmosphere, giving $196 \mathrm{mg}(95 \%)$ of carbinol 12a as a white solid $\left(\mathrm{mp} 63-65^{\circ} \mathrm{C}\right) .[\alpha]_{\mathrm{D}}^{25}=-85.4^{\circ}\left(\mathrm{c}=0.46, \mathrm{CHCl}_{3}\right) .{ }^{1} \mathrm{H}$ NMR $\left(300 \mathrm{MHz} \mathrm{CDCl}_{3}\right): \delta 7.70(\mathrm{~d}, 2 \mathrm{H}, J=7.5 \mathrm{~Hz}, \mathrm{H}-o), 7.35(\mathrm{t}, 1 \mathrm{H}, J=7.5 \mathrm{~Hz}, \mathrm{H}-p), 7.26(\mathrm{t}$, $2 \mathrm{H}, J=7.5 \mathrm{~Hz}, \mathrm{H}-m), 4.86$ (s, 1H, H-15), 4.75 (d, 2H, J=6.9 Hz, H-5a), 4.55 (d, 2H, J = $6.9 \mathrm{~Hz}, \mathrm{H}-5 \mathrm{~b}), 3.49$ (t, 1H, J = 8.5 Hz, H-7a), 3.42-3.26 (m, 3H, H-13, H-3a, H-7b), 3.25 (bs, 1H, OH), 2.97 (dd, 1H, J = 11.0, 2.8 Hz, H-3b), 2.69-2.53 (m, 2H, H-12eq, H-8), 2.402.22 (m, 4H, H-2, H-17, H-21eq, H-22eq), 2.08-1.79 (m, 4H, H-19, H-11, H-18eq, H12ax), 1.77 (s, 3H, Me-28), 1.66 (t, 1H, $J=5.7 \mathrm{~Hz}, \mathrm{H}-1), 1.62$ (t, 1H, J=4.9 Hz, H-9) 1.41 (ddd, 1H, $J=14.3,4.9,3.3 \mathrm{~Hz}, \mathrm{H}-18 \mathrm{ax}$ ), 1.23 (d, 1H, $J=9.9 \mathrm{~Hz}, \mathrm{H}-22 \mathrm{ax}$ ), 1.20 (s, 3H, Me26), 1.16 (d, 1H, J = 9.9 Hz, H-21ax), 1.12 (s, 3H, Me-24), 0.90 (s, 3H, Me-25), 0.57 (s, 3H, Me-23). ${ }^{13} \mathrm{C}$ NMR (75.4 MHz, $\mathrm{CDCl}_{3}$ ): $\delta 144.5$ (C-i), 127.7 (C-m), 127.5 (C-p), 126.7 (C-o), 96.1 (C-5), 77.2 (C-27), 74.6 (C-7), 73.2 (C-3), 62.1 (C-15), 55.2 (C-2), 54.1 (C-8), 47.0 (C-1), 43.9 (C-9), 40.9 (C-19), 40.7 (C-11), 39.2 (C-13), 37.8 (C-10), 37.3 (C-20), 37.1 (C-12), 37.1 (C-17), 34.2 (C-18), 31.3 (C-21), 30.9 (C-22), 27.0 (C-26), 26.9 (C-24), 24.7 (C-28), 23.6 (C-25), 23.1 (C-23). IR (KBr): $v_{\max }$ 3469, 1368, 1102, 2916, 1448, 1046, $698 \mathrm{~cm}^{-1}$. HRFABMS calcd. for $\mathrm{C}_{30} \mathrm{H}_{44} \mathrm{O}_{3} \mathrm{~S}_{2}+\mathrm{Na}$ : 539.2629. Found: 539.2650. 
$(1 S, 2 R, 8 R, 9 S, 13 S, 17 S)-4,6-D i o x a-14,16-d i t h i a-15-[(R)-1,2-d i p h e n y l e t h-1-y l-1-o l]-$ $10,10,20,20$-tetramethylpentacyclo-[17.1.1.1 $\left.{ }^{9,11} \cdot 0^{2,17} \cdot 0^{8,13}\right]$-docosane (12c)

To a solution of $500 \mathrm{mg}(1.02 \mathrm{mmol})$ of dodecaheterocycle 9 in anhydrous THF were added $0.54 \mathrm{~mL}(1.63 \mathrm{mmol})$ of $\mathrm{PhCH}_{2} \mathrm{MgCl} 2 \mathrm{M}$ at $-78{ }^{\circ} \mathrm{C}$ in an $\mathrm{N}_{2}$ atmosphere, giving $592 \mathrm{mg}$ $(98 \%)$ of carbinol 12c as colorless oil. $[\alpha]^{25}=+33.6\left(\mathrm{c}=0.66, \mathrm{CHCl}_{3}\right) .{ }^{1} \mathrm{H}$ NMR $(300$ $\left.\mathrm{MHz} \mathrm{CDCl}_{3}\right) \delta 7.57$ (d, 2H, J = 7.1 Hz, H-o), 7.30-7.13 (m, 3H, H-o', H-p), 7.11-7.04 (m, 5H, H-m, H-m', H-p’), 5.01 (s, 1H, H-15), 4.76 (d, 1H, J = 6.8 Hz, H-5a), 4.58 (d, 1H, J= $6.8 \mathrm{~Hz}, \mathrm{H}-5 \mathrm{~b}), 3.98$ (t, 1H, J = 7.8 Hz, H-7a), 3.66 (d, 1H, J = 14.0 Hz, H-28a), 3.58 (dd, $1 \mathrm{H}, J=11.2,8.6 \mathrm{~Hz}, \mathrm{H}-3 \mathrm{a}), 3.44$ (d, 1H, $J=14.0 \mathrm{~Hz}, \mathrm{H}-28 \mathrm{~b}), 3.39$ (q, 1H, $J=5.5 \mathrm{~Hz}, \mathrm{H}-$ 13), 3.29 (dd, 1H, J = 9.1, 2.9 Hz, H-7b), 3.10 (dd, $J=11.1,2.6$ Hz, H-3b), 2.99 (bs, 1H, $\mathrm{OH}), 2.63-2.23$ (m, 7H, H-2, H-8, H-17, H-18eq, H- 21 eq, H-22eq, H-12eq), 2.16 (m, 1-H, H-1), 2.09 (m, 1-H, H-9), 1.92-1.86 (m, 2H, H-11, H-19), 1.77 (m, 1H, H-12ax), 1.59 (m, 1H, H-18ax), 1.26 (d, 1H, $J=9.7$ Hz, H-22ax), 1.18 (s, 6H, Me-24, Me-26), 1.12 (d, 1H, $J$ $=9.9 \mathrm{~Hz}, \mathrm{H}-21 \mathrm{ax}), 0.92$ (s, 3H, Me-25), 0.74 (s, 3H, Me-23). ${ }^{13} \mathrm{C} \mathrm{NMR}(75.4 \mathrm{MHz}$, $\left.\mathrm{CDCl}_{3}\right): \delta 142.4(\mathrm{C}-i), 136.6(\mathrm{C}-i$ '), 130.8 (C-o’), 127.7 (C-m’), $127.4(\mathrm{C}-m), 127.2(\mathrm{C}-o)$, 127.0 (C-p), 126.1 (C-p’), 96.2 (C-5), 80.2 (C-27), 73.8 (C-7), 73.4 (C-3), 62.6 (C-15), 54.3 (C-13), 53.8 (C-17), 46.8 (C-11), 44.2 (C-19), 44.1 (C-28), 41.1 (C-1), 41.0 (C-9), 39.1 (C2), 38.0 (C-8), 37.7 (C-20), 37.5 (C-10), 37.4 (C-18), 34.5 (C-12), 32.0 (C-21), 31.1(C-22), 27.1 (C-24), 27.0 (C-26), 23.6 (C-25), 23.3 (C-23). IR (KBr): $v_{\max }$ 3469, 2916, 1451, 1384, 1102, 1046, $699 \mathrm{~cm}^{-1}$. LRMS (EI) m/z: $592(\mathrm{M})^{+}, 407$ (51), 395 (100), 197 (34), 135 (60), 136 (28). HRFABMS calcd. for $\mathrm{C}_{36} \mathrm{H}_{48} \mathrm{O}_{3} \mathrm{~S}_{2}+\mathrm{Na}$ : 615.2942. Found: 615.2923.

$(1 S, 2 R, 8 R, 9 S, 13 S, 17 S)-4,6-D i o x a-14,16-d i t h i a-15-[(R)-1-p h e n y l-2-p r o p e n-1-y l-1-o l]-$ $10,10,20,20$-tetramethylpentacyclo-[17.1.1.1 $\left.{ }^{9,11} \cdot 0^{2,17} \cdot 0^{8,13}\right]$-docosane (12e)

To a solution of $300 \mathrm{mg}(0.60 \mathrm{mmol})$ of dodecaheterocycle 9 in anhydrous THF were added $2.4 \mathrm{~mL}(2.4 \mathrm{mmol})$ of $\mathrm{CH}_{2}=\mathrm{CHMgBr} 1 \mathrm{M}$ at $-78{ }^{\circ} \mathrm{C}$ in an $\mathrm{N}_{2}$ atmosphere, giving $310 \mathrm{mg}$ $(98 \%)$ of carbinol 12e as colorless oil. $[\alpha]^{25}=-99.8\left(\mathrm{c}=0.53, \mathrm{CHCl}_{3}\right) .{ }^{1} \mathrm{H} \mathrm{NMR}(300$ $\mathrm{MHz}, \mathrm{CDCl}_{3}$ ): $\delta 7.68(\mathrm{~d}, 2 \mathrm{H}, J=7.5 \mathrm{~Hz}, \mathrm{H}-o$ ), 7.34-7.23 (m, 3H, H-m, H-p), 6.84 (dd, 1H, $J=17.1,10.4 \mathrm{~Hz}, \mathrm{H}-28), 5.59$ (dd, 1H, $J=17.1,1.5 \mathrm{~Hz}, \mathrm{H}-29 \mathrm{a}), 5.37$ (dd, 1H, $J=10.4,1.5$ Hz, H-29b), 4.93 (s, 1H, H-15), 4.82 (d, 1H, J = 6.7 Hz, H-5a) 4.60 (d, 1H, J = 6.7 Hz, H- 
5b), 3.96 (t, 1H, $J=7.8, \mathrm{H}-7 \mathrm{a}), 3.46-3.27$ (m, 4H, OH, H-3a, H-7b, H-13), 3.10 (dd, 1H, $J$ = 10.9, 3.1 Hz, H-3b), 2.65-2.50 (m, 2H, H-8, H-12), 2.41-2.23 (m, 4H, H-21eq, H-22eq, H-17, H-13), 2.07-1.72 (m, 7H, H-12ax, H-11, H-1, H-9, H-18eq, H-19, H-2), 1.65 (m, 1H, H-22ax), 1.42 (m, 1H, H-18ax), 1.23 (d, 1H, $J=9.9$ Hz, H-21ax), 1.19 (d, 1H, $J=9.9$ Hz, H-22ax), 1.21 (s, 3H, Me-26), 1.19 (s, 3H, Me-24), 0.89 (s, 3H, Me-25), 0.58 (s, 3H, Me23). ${ }^{13} \mathrm{C}$ NMR (75.4 MHz, $\mathrm{CDCl}_{3}$ ): $\delta 143.1(\mathrm{C}-i), 139.8$ (C-28), 127.7 (C-m), 127.5 (C-p), 126.9 (C-o), 115.8 (C-29), 95.9 (C-5), 78.7 (C-27), 74.6 (C-3), 72.9 (C-7), 61.3 (C-15), 55.2 (C-13), 53.9 (C-17), 46.9 (C-11), 44.0 (C-19), 40.8 (C-1), 40.6 (C-9), 38.7 (C-2), 37.7 (C-8), 37.3 (C-10), 37.1 (C-20), 36.8 (C-18), 34.3 (C-12), 31.2 (C-21), 31.1 (C-22), 27.0 (24), 26.9 (C-26), 23.5 (C-25), 23.0 (C-23). IR (KBr): $v_{\max }$ 3421, 2916, 1449, 1340, 1102, 1047, $699 \mathrm{~cm}^{-1}$. LRMS (EI) m/z: $528(\mathrm{M})^{+}, 395$ (100), 197 (28), 135 (62), 133 (37), 93 (25). HRFABMS calcd. for $\mathrm{C}_{31} \mathrm{H}_{44} \mathrm{O}_{3} \mathrm{~S}_{2}+\mathrm{Na}$ : 551.2629. Found: 551.2645

\section{$(1 S, 2 R, 8 R, 9 S, 13 S, 17 S)-4,6-D i o x a-14,16-d i t h i a-15-[(R)-1-p h e n y l-3-m e t h y l-2-b u t e n-1-$ yl-1-ol]-10,10,20,20-tetramethylpentacyclo-[17.1.1.1 $\left.{ }^{9,11} \cdot 0^{2,17} \cdot 0^{8,13}\right]$-docosane (12f)}

To a solution of $300 \mathrm{mg}(0.60 \mathrm{mmol})$ of dodecaheterocycle 9 in anhydrous THF were added $4.8 \mathrm{~mL}$ (2.4 mmol) of $\mathrm{CH}_{2}=\mathrm{C}\left(\mathrm{CH}_{3}\right) \mathrm{CH}_{2} \mathrm{MgBr} 0.6 \mathrm{M}$ at $-78{ }^{\circ} \mathrm{C}$ in an $\mathrm{N}_{2}$ atmosphere, giving $319 \mathrm{mg}(98 \%)$ of carbinol $\mathbf{1 2 f}$ as colorless oil. $[\alpha]^{25}{ }_{\mathrm{D}}=-86.6\left(\mathrm{c}=1.0, \mathrm{CHCl}_{3}\right) .{ }^{1} \mathrm{H} \mathrm{NMR}$ (300 MHz, CDCl $): \delta 7.65$ (d, 2H, $J=7.3 \mathrm{~Hz}, \mathrm{H}-o$ ), 7.33 (t, 2H, $J=7.3 \mathrm{~Hz}, \mathrm{H}-m$ ), 7.26 (t, $1 \mathrm{H}, J=7.3 \mathrm{~Hz}, \mathrm{H}-p), 4.87$ (s, 1H, H-15), 4.80 (s, 1H, H-30a), 4.74 (d, 1H, $J=7.3 \mathrm{~Hz}, \mathrm{H}-$ 5a), 4.71 (s, 1H, H-30b), 4.56 (d, 1H, $J=7.3 \mathrm{~Hz}, \mathrm{H}-5 \mathrm{~b}), 3.96$ (t, 1H, $J=8.3 \mathrm{~Hz}, \mathrm{H}-7 \mathrm{a}$ ), 3.52 (bt, $1 \mathrm{H}, J=11.5 \mathrm{~Hz}, \mathrm{H}-3 \mathrm{a}$ ), 3.40-3.32 (m, 2H, H-13, H-7b), 3.21 (d, 1H, $J=14.6 \mathrm{~Hz}$, H-28a), 3.09 (bs, 1H, OH), 3.05 (dd, 1H, $J=2.6,3.1 \mathrm{~Hz}, \mathrm{H}-3 \mathrm{~b}$ ), 2.76 (d, 1H, $J=14.6 \mathrm{~Hz}$, H-28b), 2.62-2.50 (m, 2H, H-12eq, H-8), 2.41-2.19 (m, 5H, H-21 eq, H-22eq, H-2, H-17, H$18 \mathrm{eq}), 2.12(\mathrm{td}, 1 \mathrm{H}, J=5.7,2.1 \mathrm{~Hz}, \mathrm{H}-9) 2.01$ (td, $1 \mathrm{H}, J=5.7,2.1 \mathrm{~Hz}, \mathrm{H}-1), 1.93-1.85$ (m, 2H, H-19, H-11), 1.81-1.70 (m, 1H, H-18ax), 1.47 (s, 3H, Me-31) 1.23 (d, 1H, J = 9.4 Hz, H-22ax), 1.18 (s, 3H, Me-26), 1.16 (s, 3H, Me-24), 1.14 (d, 1H, J = 10.4 Hz, H-21ax), 0.91 (s, 3H, Me-25), 0.71 (s, 3H, Me-23). ${ }^{13} \mathrm{C}$ NMR (75.4 MHz, $\mathrm{CDCl}_{3}$ ): $\delta 142.5$ (C-29), 141.9 (C-i), 127.4 (C-o), 127.1 (C-p), 127.0 (C-m), 115.5 (C-30), 96.1 (C-5), 78.9 (C-29), 79.9 (C-7), 73.3 (C-3), 62.7 (C-15), 54.3 (C-2), 53.8 (C-8), 46.8 (C-1), 45.3 (C-30), 43.8 (C-9), 
41.0 (C-19), 40.9 (C-11), 39.1 (C-13), 37.9 (C-10), 37.5 (C-12), 37.3 (C-20), 37.1 (C-17), 34.6 (C-18), 31.9 (C-21), 30.9 (C-22), 27.0 (C-26), 26.9 (C-24), 24.3 (C-31), 23.6 (C-25), 23.2 (C-23). IR (Film): $v_{\max }$ 3435, 2917, 1449, 1366, 1102, 1046, $699 \mathrm{~cm}^{-1}$. LRMS (EI) m/z: $557(\mathrm{M}+1)^{+}, 395$ (100), 135 (72), 93 (32), 197 (25). HRFABMS calcd. for $\mathrm{C}_{33} \mathrm{H}_{48} \mathrm{O}_{3} \mathrm{~S}_{2}+\mathrm{Na}: 579.2942$. Found: 579.2942.

$(1 S, 2 R, 8 R, 9 S, 13 S, 17 S)-4,6-D i o x a-14,16-d i t h i a-15-[(R)-1-p h e n y l-2-b u t y n-1-y l-1-o l]-$ $10,10,20,20$-tetramethylpentacyclo-[17.1.1.1 $\left.{ }^{9,11} \cdot 0^{2,17} \cdot 0^{8,13}\right]$-docosane $(12 \mathrm{~g})$

To a solution of $300 \mathrm{mg}(0.60 \mathrm{mmol})$ of dodecaheterocycle $\mathbf{9}$ in anhydrous THF were added $0.54 \mathrm{~mL}$ (1.63 mmol) of $\mathrm{CH}_{3} \mathrm{CCMgBr} 0.5 \mathrm{M}$ at $-78{ }^{\circ} \mathrm{C}$ in an $\mathrm{N}_{2}$ atmosphere, giving $308 \mathrm{mg}$ $(95 \%)$ of carbinol $12 \mathrm{~g}$ as a white solid $\left(\mathrm{mp} 73-75^{\circ} \mathrm{C}\right) \cdot[\alpha]_{\mathrm{D}}^{25}=-55.2^{\circ}\left(\mathrm{c}=0.98, \mathrm{CHCl}_{3}\right)$. ${ }^{1} \mathrm{H}$ NMR (300 MHz, $\mathrm{CDCl}_{3}$ ): $\delta 7.82(\mathrm{~d}, 2 \mathrm{H}, J=7.3 \mathrm{~Hz}, \mathrm{H}-o$ ), 7.38-7.26 (m, 3H, H-m, H- $p$ ), 4.78 (s, 1H, H-15), 4.76 (d, 1H, $J=6.7 \mathrm{~Hz}, \mathrm{H}-5 \mathrm{a}$ ), 4.58 (d, 1H, $J=6.7 \mathrm{~Hz}, \mathrm{H}-5 \mathrm{~b}$ ), 4.99 (s, 1H, OH), 3.96 (m, 1H, H-7a), 3.45-3.38 (m, 2H, H-3a, H-13), 3.31 (dd, 1H, $J=9.3,3.1 \mathrm{~Hz}$, H-7b), 3.19 (dd, 1H, $J=10.9,3.1$ Hz H-3b), 2.66-2.45 (m, 2H, H-12eq, H-8), 2.39-2.22 (m, 4H, H-2, H-21eq, H-22eq, H-17), 2.1-1.74 (m, 6H, H-18eq, H-12ax, H-1, H-19, H-11, H9), 1.96 (s, 3H, H-30), 1.41-1.38 (m, 1H, H-18ax), 1.21 (d, $J=9.9$ Hz, 1H, H-22ax), 1.20 (s, 3H, Me-26), 1.12 (s, 3H, Me-24), 1.10 (d, 1H, J = 9.9 Hz, H-21ax), 0.91 (s, 3H, Me-25), 0.61 (s, 3H, Me-23). ${ }^{13} \mathrm{C}$ NMR (75.4 MHz, $\mathrm{CDCl}_{3}$ ): $\delta 141.4(\mathrm{C}-i), 128.1(\mathrm{C}-p), 127.5$ (Cm), 127.2 (C-o), 95.6 (C-5), 83.2 (C-28), 80.1 (C-29), 75.7 (C-27), 74.2 (C-7), 72.8 (C-3), 62.9 (C-15), 54.7 (C-2), 53.8 (C-8), 47.2 (C-1), 44.4 (C-9), 41.1 (C-19), 40.9 (C-11), 38.5 (C-13), 37.9 (C-10), 37.4 (C-20), 37.2 (C-17,12), 34.5 (C-18), 31.4 (C-21, 22), 27.1 (C-26), 26.9 (C-24), 23.6 (C-25), 23.1 (C-23). IR (KBr): $v_{\max } 3435,2916,1450,1385,1102,1046$, $699 \mathrm{~cm}^{-1}$. LRMS (EI) m/z: $563(\mathrm{M})^{+}, 523$ (47), 395 (100), 136 (43), 135 (100), 136 (43). HRFABMS calcd. for $\mathrm{C}_{32} \mathrm{H}_{44} \mathrm{O}_{3} \mathrm{~S}_{2}+\mathrm{Na}: 563.2629$. Found: 563.2626.

$(1 S, 2 R, 8 R, 9 S, 13 S, 17 S)$-4,6-Dioxa-14,16-dithia-15-[(R)-1,2-diphenyl-2-propyn-1-yl-1ol]-10,10,20,20-tetramethylpentacyclo-[17.1.1.1 $\left.{ }^{9,11} \cdot 0^{2,17} \cdot 0^{8,13}\right]$-docosane (12h)

To a solution of $300 \mathrm{mg}(0.60 \mathrm{mmol})$ of dodecaheterocycle 9 in anhydrous THF were added $2.4 \mathrm{~mL}$ (2.4 mmol) of $\mathrm{PhCCMgBr} 1 \mathrm{M}$ at $-78{ }^{\circ} \mathrm{C}$ in an $\mathrm{N}_{2}$ atmosphere, giving $354 \mathrm{mg}$ (98\%) 
of carbinol 12h as colorless oil. $[\alpha]^{25}=-140.6\left(\mathrm{c}=0.33 \mathrm{CHCl}_{3}\right) .{ }^{1} \mathrm{H}$ NMR $(300 \mathrm{MHz}$, $\left.\mathrm{CDCl}_{3}\right): \delta 7.89(\mathrm{~d}, 2 \mathrm{H}, J=7.8 \mathrm{~Hz}, \mathrm{H}-o$ ), 7.53 (d, 2H, $J=7.3 \mathrm{~Hz}, \mathrm{H}-o$ '), 7.42-7.29 (m, 6H, H- $\left.p, \mathrm{H}-p^{\prime}, \mathrm{H}-m, \mathrm{H}-m^{\prime}\right), 4.91$ (s, 1H, H-15), 4.75 (d, 1H, J=6.8 Hz, H-5a), 4.57 (d, 1H, J= $6.8 \mathrm{~Hz}, \mathrm{H}-5 \mathrm{~b}), 4.17$ (bs, 1H, OH), 3.97 (dd, 1H, J = 9.4, $8.8 \mathrm{~Hz}, \mathrm{H}-7 \mathrm{a})$ 3.51-3.40 (m, 2H, H-13, H-3a), 3.33 (dd, 1H, J=9.9, 3.1 Hz, H-7b), 3.23 (dd, 1H, J=10.9, 3.1 Hz, H-3b), 2.69-2.63 (m, 2H, H-12eq, H-8), 2.38-2.24 (m, 4H, H-2, H-17, H-22eq, H-21 eq), 2.15-2.0 (m, 3H, H-1, H-9, H-18eq), 1.95-1.77 (m, 3H, H-12ax, H-19, H-11), 1.41 (m, 1H, H-18ax), 1.22 (d, 1H, J=9.9 Hz, H-22ax), 1.2 (s, 3H, Me-26), 1.13 (d, J = 9.9 Hz, H-21ax), 1.12 (s, 3H, Me-24). 0.87 (s, 3H, Me-25), 0.58 (t, 3H, Me-23). ${ }^{13} \mathrm{C}$ NMR (75.4 MHz, $\left.\mathrm{CDCl}_{3}\right): \delta$ 140.9 (C-i), 131.9 (C-o’), 128.6 (C-m), 128.3 (C-p), 128.2 (C-m’), 127.7 (C-p’), 127.1 (Co), 122.3 (C-i’), 95.7 (C-5), 90.0 (C-29), 86.7 (C-28), 76.1 (C-27), 74.4 (C-7), 72.8 (C-3), 62.9 (C-15), 54.8 (C-2), 53.8 (C-8), 47.0 (C-1), 44.3 (C-9), 40.9 (C-19), 40.8 (C-11), 38.7 (C-13), 37.9 (C-10), 37.4 (C-20), 37.2 (C-12), 37.1 (C-17), 34.4 (C-18), 31.3 (C-22, C-21), 27.0 (C-26), 26.9 (C-24), 23.6 (C-25), 23.1 (C-23). IR (Film): $v_{\max }$ 3421, 2982, 1449, 1385, 1102, 1047, $693 \mathrm{~cm}^{-1}$. LRMS (EI) m/z: $602(\mathrm{M})^{+}, 395$ (100), 197 (30), 135 (75), 93 (28). HRFABMS calcd. for $\mathrm{C}_{37} \mathrm{H}_{46} \mathrm{O}_{3} \mathrm{~S}_{2}+\mathrm{Na}$ : 625.2786. Found: 625.2781.

\section{$(1 S, 2 R, 8 R, 9 S, 13 S, 17 S)-4,6-D i o x a-14,16-d i t h i a-15-[(R)-p h e n y l m e t h a n o l]-10,10,20,20-$ tetramethylpentacyclo-[17.1.1.1 $\left.1^{9,11} \cdot 0^{2,17} \cdot 0^{8,13}\right]$-docosane $(12 j)$}

A solution of $300 \mathrm{mg}(0.60 \mathrm{mmol})$ of dodecaheterocycle 9 in $5 \mathrm{~mL}$ of dry THF was added to a well-stirred and cooled $\left(-78{ }^{\circ} \mathrm{C}\right)$ suspension of $90.8 \mathrm{mg}(2.4 \mathrm{mmol})$ of $\mathrm{LiAlH}_{4}$. The mixture was further stirred for $4 \mathrm{~h}$ at $-78{ }^{\circ} \mathrm{C}$. After the addition of $50 \mathrm{~mL}$ of ethyl ether, the reaction was quenched by slow successive addition of small pieces of ice and $1 \mathrm{~mL}$ of cold water. The organic layer was washed with brine $(2 \times 25 \mathrm{~mL})$, dried over $\mathrm{Na}_{2} \mathrm{SO}_{4}$ and evaporated to dryness, yielding $286 \mathrm{mg}$ (95\%) of carbinol 12j as a white solid (mp 138-140 $\left.{ }^{\circ} \mathrm{C}\right) .[\alpha]_{\mathrm{D}}^{25}=+71.4\left(\mathrm{c}=0.21, \mathrm{CHCl}_{3}\right) .{ }^{1} \mathrm{H}$ NMR $\left(300 \mathrm{MHz}, \mathrm{CDCl}_{3}\right): \delta 7.46(\mathrm{~d}, 2 \mathrm{H}, J=8.2$ $\mathrm{Hz}, \mathrm{H}-o$ ), 7.39-7.31 (m, 3H, H-p, H-m), 4.63 (d, 1H, J=6.6 Hz, H-5a), 4.63 (d, 1H, J = 6.6 Hz, H-5b), 4.53 (d, 1H, J=9.3 Hz, H-27), 4.44 (d, 1H, J = 9.3 Hz, H-15), 3.79-3.63 (m, 5H, H-7a, H-7b, H-3a, H-13, OH), 3.32 (dd, 1H, J = 3.3, 9.3 Hz, H-3b), 3.00 (bq, 1H, $J=$ 7.7 Hz, H-17), 2.63 (tt, 1H, J = 2.2, 10.4 Hz, H-12eq), 2.49-2.41 (m, 1H, H-22eq), 2.38- 
2.22 (m, 3H, H-21eq, H-8, H-18eq), 2.11-1.49 (m, 5H, H-12ax, H-2, H-1, H-11, H-9), 1.85 (ddd, 1H, $J=6.6,3.8,2.5$ Hz, H-19), 1.62 (m, 1H, H-18ax), 1.23 (s, 3H, Me-26), 1.17 (s, $3 \mathrm{H}, \mathrm{Me}-24), 1.13$ (d, 1H, J=9.9 Hz, H-22ax), 1.01 (s, 3H, Me-25), (d, 1H, J = 9.9 Hz, H21ax), 0.98 (s, 3H, Me-23). ${ }^{13} \mathrm{C}$ NMR (75.4 MHz, $\left.\mathrm{CDCl}_{3}\right): \delta 140.6(\mathrm{C}-i), 128.2(\mathrm{C}-p)$, 128.1 (C-m), 127.3 (C-o), 94.1 (C-5), 75.8 (C-27), 71.2 (C-3), 70.7 (C-7), 59.5 (C-15), 51.4 (C-8,2), 47.3 (C-1), 46.2 (C-9), 41.9 (C-19), 41.7 (C-11), 39.0 (C-12), 38.4 (C-10), 38.2 (C20), 36.0 (C-13), 35.5 (C-17), 34.1 (C-22), 33.9 (C-21), 27.7 (C-26), 27.6 (24), 24.4 (C-25), 24.1 (C-23). IR (KBr): $v_{\max } 2918,1448,1048,700 \mathrm{~cm}^{-1}$. HRFABMS calcd. for $\mathrm{C}_{29} \mathrm{H}_{42} \mathrm{O}_{3} \mathrm{~S}_{2}+$ Na: 525.2427. Found: 525.2485. EA calcd. for $\mathrm{C}_{29} \mathrm{H}_{42} \mathrm{O}_{3} \mathrm{~S}_{2}$ : C, 69.45; H, 8.42. Found: C, $69.45 ; \mathrm{H}, 8.23$.

\section{$(1 S, 2 R, 8 R, 9 S, 13 S, 17 S)-4,6-D i o x a-14,16-d i t h i a-15-[(S)$-phenylmethanol]-10,10,20,20- tetramethylpentacyclo-[17.1.1.1 $\left.{ }^{9,11} \cdot 0^{2,17} \cdot 0^{8,13}\right]$-docosane $(13 j)$}

To a cooled $\left(-78^{\circ} \mathrm{C}\right)$ solution of $300 \mathrm{mg}(0.75 \mathrm{mmol})$ of dodecaheterocycle $8 \mathbf{a}$ in $5 \mathrm{~mL}$ of anhydrous THF were added $1.4 \mathrm{~mL}(1.4 \mathrm{mmol})$ of sec-BuLi $1.4 \mathrm{M}$ in THF, and the resulting mixture was stirred under an $\mathrm{N}_{2}$ atmosphere at the same temperature for 20 min. Then, $160 \mathrm{mg}(1.4 \mathrm{mmol})$ of benzaldehyde were added and the mixture was stirred at the above temperature during $1.5 \mathrm{~h}$. The reaction mixture was quenched with a saturated solution of $\mathrm{NH}_{4} \mathrm{Cl}$, extracted with $\mathrm{CH}_{2} \mathrm{Cl}_{2}$, washed with water $(3 \times 10 \mathrm{~mL})$, dried with anhydrous $\mathrm{Na}_{2} \mathrm{SO}_{4}$ and evaporated to dryness, giving $369 \mathrm{mg}(97 \%)$ of a mixture of carbinols 12j and 13j (35:65) as colorless oil. The mixture was filtered through a short column chromatography (silica gel 230-400; hexane/EtOAc 49:1), affording 229 mg (62 \%) of $\mathbf{1 3} \mathbf{j}$ as colorless oil and $129 \mathrm{mg}(35 \%)$ of $\mathbf{1 2} \mathbf{j}$ as white solid (mp 138-140 ${ }^{\circ} \mathrm{C}$ ). Data for 13j: $[\alpha]^{25}=-45.8\left(\mathrm{c}=0.26, \mathrm{CHCl}_{3}\right) .{ }^{1} \mathrm{H} \mathrm{NMR}\left(300 \mathrm{MHz}, \mathrm{CDCl}_{3}\right): \delta 7.48(\mathrm{~d}, 2 \mathrm{H}, J=7.4$ $\mathrm{Hz}, \mathrm{H}-o$ ), 7.36 (t, 1H, J=7.4 Hz, H-p), 7.28 (t, 2H, J=7.4 Hz, H-m), 5.01 (s, 2H, H-15, H27), 4.67 (d, 1H, $J=6.6 \mathrm{~Hz}, \mathrm{H}-5 \mathrm{a}), 4.54$ (d, 1H, $J=6.6 \mathrm{~Hz}, \mathrm{H}-5 \mathrm{~b}), 3.90$ (q, 1H, $J=9.3 \mathrm{~Hz}$, H-3a), 3.54-3.42 (m, 4H, H-7a, H-7b, H-3b, H-13), 3.36 (dd, 1H, J = 2.7, 9.3 Hz, H-17), 3.02 (dd, 1H, J = 11.0, 2.7 Hz, H-7b), 2.61-2.51 (m, 2H, H-2, H-8), 2.49-2.41 (m, 2H, H22eq, H-21eq), 2.25-2.16 (m, 2H, H-12eq, H-18eq), 2.08-1.99 (m, 1H, H-11), 1.95-1.90 (m, 1H, H-1), 1.88-1.81 (m, 1H, H-9), 1.79-1.76 (m, 1H, H-19), 1.66-1.64 (m, 1H, H- 
18ax), 1.61-1.59 (m, 1H, H-12ax), 1.19 (s, 3H, Me-26), 1.15 (d, 1H, J = 8.8 Hz, H-22ax), 1.15 (s, 3H, Me-24), 0.93 (s, 3H, Me-25), 0.73 (d, 1H, J = 8.2 Hz, H-21ax), 0.64 (s, 3H,

Me-23). ${ }^{13} \mathrm{C}$ NMR (75.4 MHz, $\left.\mathrm{CDCl}_{3}\right): \delta 141.8(\mathrm{C}-i), 128.1$ (C-p), $127.9(\mathrm{C}-m), 126.5(\mathrm{C}-$ o), 95.6 (C-5), 76.1 (C-27), 74.0 (C-3), 72.5 (C-7), 58.1 (C-15), 54.6 (C-8, ), 53.6 (C-2), 46.9 (C-1), 44.5 (C-9), 41.1 (C-19,C-11), 38.9 (C-13, C-17), 37.9 (C-10, C-20), 37.6 (C-2), 37.5 (C-8), 36.9 (C-18), 35.0 (C-12), 32.2 (C-22), 31.9 (C-21), 27.1 (C-26, C-24), 23.7 (25), 23.3 (C-23). IR (Film): $v_{\max }$ 2920, 1440, 1052, $708 \mathrm{~cm}^{-1}$. HRFABMS calcd. for $\mathrm{C}_{29} \mathrm{H}_{42} \mathrm{O}_{3} \mathrm{~S}_{2}+\mathrm{Na}: 525.2427$. Found: 525.2462.

\section{General procedure for the addition of lithium reagents to dodecaheterocycle 9}

To a solution of dodecaheterocycle 9 (1 equiv.) in anhydrous THF was added the organolithium reagent (4 equiv.) at $-78{ }^{\circ} \mathrm{C}$ under a nitrogen atmosphere. After stirring for 2 $\mathrm{h}$ at the same temperature, the reaction mixture was allowed to warm up to room temperature and further stirred for $2 \mathrm{~h}$. The reaction mixture was quenched with a saturated solution of ammonium chloride, the THF was eliminated by evaporation and the remaining emulsion was extracted with ethyl ether. The organic layer was washed with a saturated solution of ammonium chloride, dried over anhydrous $\mathrm{Na}_{2} \mathrm{SO}_{4}$ and concentrated to dryness, giving the corresponding pure diastereoisomer $\mathbf{1 2}$ as colorless oil.

\section{$(1 S, 2 R, 8 R, 9 S, 13 S, 17 S)-4,6-D i o x a-14,16-d i t h i a-15-[(R)-p h e n y l p e n t-1-y l-1-o l]-$} $10,10,20,20$-tetramethylpentacyclo-[17.1.1.1 $\left.{ }^{9,11} \cdot 0^{2,17} \cdot 0^{8,13}\right]$-docosane (12i)

To a solution of $300 \mathrm{mg}(0.60 \mathrm{mmol})$ of dodecaheterocycle 9 in anhydrous THF were added $0.24 \mathrm{~mL}(2.4 \mathrm{mmol})$ of $n$-BuLi $10 \mathrm{M}$ at $-78^{\circ} \mathrm{C}$ in an $\mathrm{N}_{2}$ atmosphere, giving $328 \mathrm{mg}(98 \%)$ of carbinol 12i as colorless oil. $[\alpha]^{25}=-48.3\left(\mathrm{c}=0.53, \mathrm{CHCl}_{3}\right) .{ }^{1} \mathrm{H}$ NMR $(300 \mathrm{MHz}$, $\left.\mathrm{CDCl}_{3}\right): \delta 7.66(\mathrm{~d}, 2 \mathrm{H}, J=8.3 \mathrm{~Hz}, \mathrm{H}-o), 7.35(\mathrm{t}, 2 \mathrm{H}, J=8.3 \mathrm{~Hz}, \mathrm{H}-m), 7.25(\mathrm{t}, 1 \mathrm{H}, J=8.3$ Hz, H-p), 4.84 (s, 1H, H-15), 4.73 (d, 1H, J = 7.3, H-5a), 4.54 (d, 1H, J = 7.3, H-5b), 3.94 (t, 1H, $J=8.3 \mathrm{~Hz}, \mathrm{H}-7 \mathrm{a}), 3.39$ (t, 1H, $J=10.6 \mathrm{~Hz}, \mathrm{H}-3 \mathrm{a}) 3.34-3.23$ (m, 2H, H-7b, H-13), 3.01 (bs, 1H, OH), 2.86 (dd, 1H, $J=10.6,2.0$ Hz, H-3b), 2.64-2.46 (m, 3H, H-17, H-12eq, H-8), 2.41-2.24 (m, 3H, H-22eq, H-2, H-21eq), 2.16-1.99 (m, 3H, H-18eq, H-1, H-9), 1.94- 
1.89 (m, 1H, H-11), 1.82-1.74 (m, 3H, H-12ax, H-19, H-28), 1.49-1.42 (m, 1H, H-18ax), 1.37-1.26 (m, 3H, H-30, Me-29, H-21ax), 1.20 (d, 1H, J = 7.3 Hz, H-22ax), 1.18 (s, 3H, Me-26), 1.14 (s, 3H, Me-24). 0.90 (s, 3H, Me-25), 0.86 (t, 3H, J = 7.3 Hz, Me-31), 0.61 (s, 3H, Me-23). ${ }^{13} \mathrm{C}$ NMR (75.4 MHz, $\left.\mathrm{CDCl}_{3}\right): \delta 142.2(\mathrm{C}-i), 127.6(\mathrm{C}-o), 127.2(\mathrm{C}-m), 127.1$ (C-p), 96.1 (C-5), 79.5 (C-27), 74.3 (C-7), 73.2 (C-3), 62.8 (C-15), 54.7 (C-2), 54.0 (C-8), 46.9 (C-1), 43.7 (C-9), 40.8 (C-19), 40.7 (C-11), 39.3 (C-13), 37.8 (C-10), 37.3 (C-12), 37.3 (C-20), 37.1 (C-17), 36.0 (C-28), 34.0 (C-18), 31.5 (C-22), 30.8 (C-21), 27.0 (C-26, C-24), 25.5 (C-29), 23.5 (C-25), 23.1 (C-23, C-30), 14.1 (C-31). IR (Film) $v_{\max }$ 3502, 2919, 1452, 1384, 1103, 1046, $699 \mathrm{~cm}^{-1}$. HRFABMS calcd. for $\mathrm{C}_{33} \mathrm{H}_{50} \mathrm{O}_{3} \mathrm{~S}_{2}+\mathrm{Na}: 581.3099$ Found: 581.3080 .

General procedure for the hydrolysis of carbinols $12 \mathrm{e}, 12 \mathrm{j}$ and $12 \mathrm{~d}$ and successive reduction of the corresponding aldehydes to obtain 1,2-propanediols 16a, 16c and 16e

Carbinol 12 (1 equiv.) in $5 \mathrm{~mL}$ of $\mathrm{CH}_{3} \mathrm{CN}$ was treated with 2 equiv of NCS and 2 equiv of $\mathrm{AgNO}_{3}$ in $5 \mathrm{~mL}$ of $\mathrm{CH}_{3} \mathrm{CN}-\mathrm{H}_{2} \mathrm{O}(4: 1)$ at $0-4{ }^{\circ} \mathrm{C}$ during $1-5 \mathrm{~min}$. The work-up was carried out by successive addition of solutions of $\mathrm{NaCl}, \mathrm{Na}_{2} \mathrm{SO}_{3}$ and $\mathrm{Na}_{2} \mathrm{CO}_{3}$ (1 mL each), the white precipitated was filtered, and the filtrate was extracted with a mixture of hexane/methylene chloride (1:1). The organic layer was dried with anhydrous $\mathrm{Na}_{2} \mathrm{SO}_{4}$, filtrated and concentrated to dryness, giving a crude yellowish oil, whose ${ }^{1} \mathrm{H}$ NMR spectrum showed the presence of the corresponding aldehyde. The mixture was treated with $\mathrm{NaBH}_{4}$ in a mixture of acetonitrile/water (1:1), stirred at room temperature for $2 \mathrm{~h}$, followed by addition of $10 \mathrm{~mL}$ of hot water, the stirring continued for $20 \mathrm{~min}$ and the mixture was extracted with ethyl ether. The organic layer was dried with anhydrous $\mathrm{Na}_{2} \mathrm{SO}_{4}$, concentrated to dryness and the yellowish oily residue was flash chromatographed using a mixture of hexane/ethyl acetate (7:3) as eluent, giving hydroxythiol and the corresponding diol 16.

\section{(R)-2-Phenylpropane-1,2-diol (16a)}


The crude reaction mixture obtained from the hydrolysis of $400 \mathrm{mg}(0.77 \mathrm{mmol})$ of adduct 12a, was treated with $\mathrm{NaBH}_{4}$ as described above. After column chromatography were obtained $94 \mathrm{mg}(71 \%)$ of diol 16a as colorless oil. $[\alpha]^{23}=-5.7(\mathrm{c}=0.50$, EtOH $)$; Lit. $[\alpha]^{24}=$ -5.8 (c, 0.17, EtOH)). ${ }^{1} \mathrm{H}$ NMR (300 MHz, $\left.\mathrm{CDCl}_{3}\right) \delta$ 7.44-7.27 (m, 5H, Ar), 3.49 (d, 1H, J $=11.1 \mathrm{~Hz}, \mathrm{H}-1 \mathrm{a}), 3.42$ (d, 1H, $J=11.1 \mathrm{~Hz}, \mathrm{H}-1 \mathrm{~b}), 2.38$ (bs, 1H, OH), 2.01 (bs, 1H, OH), 1.53 (s, 3H, Me-3).

\section{(R)-2,3-Diphenylpropane-1,2-diol (16c)}

The crude reaction mixture obtained from the hydrolysis of $738 \mathrm{mg}(1.24 \mathrm{mmol})$ of adduct 12d was treated with $\mathrm{NaBH}_{4}$ as described above. After purification by column chromatography were obtained $220 \mathrm{mg}(78 \%)$ of diol $\mathbf{1 6 c}$ as colorless oil. $[\alpha]_{\mathrm{D}}^{25}=+59.5(\mathrm{c}$ $=0.25, \mathrm{EtOH}) .{ }^{1} \mathrm{H}$ NMR $\left(300 \mathrm{MHz}, \mathrm{CDCl}_{3}\right) \delta 7.34-7.16(\mathrm{~m}, 8 \mathrm{H}, \mathrm{Ar}), 6.65-6.62(\mathrm{~m}, 2 \mathrm{H}$, Ar), 3.83 (d, 1H, $J=10.9$ Hz, H-1a), 3.78 (d, 1H, $J=10.9$ Hz, H-1b), 3.19 (d, 1H, J = 13.5 Hz, H-3a), 3.19 (d, 1H, J = 13.5 Hz, H-3b), 2.53 (bs, 1H, OH) 1.81 (bs, 1H, OH).

\section{(R)-2-Phenyl-3-butene-1,2-diol (16e)}

The crude reaction mixture obtained from the hydrolysis of $305 \mathrm{mg}(0.57 \mathrm{mmol})$ of adduct 12e was treated with $\mathrm{NaBH}_{4}$ as described above. After column chromatography were obtained $76 \mathrm{mg}(80 \%)$ of diol 16e as colorless oil. $[\alpha]_{\mathrm{D}}^{25}=+35.5(\mathrm{c}=0.20$, EtOH). Lit. $[\alpha]_{\mathrm{D}}^{25}=+41.2(\mathrm{c}=.2, \mathrm{EtOH}) .{ }^{1} \mathrm{H}$ NMR $\left(300 \mathrm{MHz}, \mathrm{CDCl}_{3}\right) \delta$ 7.50-7.28 (m, 5H, Ar), 6.18 (dd, $2 \mathrm{H}, J=17.3,10.7 \mathrm{~Hz}, \mathrm{H}-3), 5.42$ (d, 1H, $J=17.3 \mathrm{~Hz}, \mathrm{H}-4 \mathrm{a}), 5.32$ (d, $J=10.7 \mathrm{~Hz}, \mathrm{H}-$ 4b), 3.83 (part A of an AB system, 1H, H-1a), 3.81 (part B of an AB system, 1H, H-1b), 2.80 (bs, 1H, OH), 1.60 (bs, 1H, OH). 
Table 1. Crystal data for dodecaheterocycle $\mathbf{9}$ and adduct $\mathbf{1 2 j}$

\section{A. Crystal parameters}

Empirical formula
Formula weight
Crystal system
Space group
Crystal size $(\mathrm{mm})$
Crystal color
Unit cell data
a $(\AA)$
b $(\AA)$
c $(\AA)$
Volume $\left(\AA^{3}\right)$
$Z$
$\rho\left(\mathrm{mg} / \mathrm{mm}^{3}\right)$
$\mathrm{F}(000)\left(\mathrm{e}^{-}\right)$

\section{B. Data collection parameters}

Reflections collected/unique

Absortion coefficient $\left(\mathrm{mm}^{-1}\right)$
Temperature $(\mathrm{K})$
Wavelength $(\AA)$
Theta range $(\mathrm{deg})$
Limiting indices

\section{Structure Refinement}

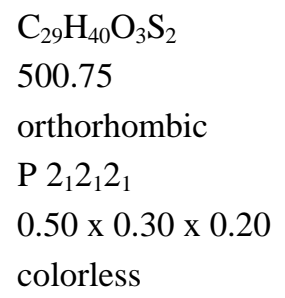

1080
$\mathrm{C}_{29} \mathrm{H}_{42} \mathrm{O}_{3} \mathrm{~S}_{2}$

502.75

orthorhombic

P $22_{1} 2_{1}$

$0.40 \times 0.44 \times 0.46$

colorless

6.5094(5)

11.567(1)

36.831(3)

2773.2(4)

4

1.20

1088

$\begin{array}{ll}1.98 & 0.219 \\ 298(2) & 297(2) \\ 1.54184 & 0.71073 \\ 4.21 \text { to } 54.94 & 1.11 \text { to } 22.74 \\ 0 \leq \mathrm{h} \leq 12, & -7 \leq \mathrm{h} \leq 7, \\ 0 \leq \mathrm{k} \leq 13, & 0 \leq \mathrm{k} \leq 12, \\ 0 \leq 1 \leq 19 & 0 \leq 1 \leq 40 \\ 2026 / 1949 & 3028 / 2755\end{array}$

Full-matrix ls on $\mathrm{F}^{2}$

Full-matrix ls on $\mathrm{F}^{2}$

1942/ 0 / 308

2546 / 1 / 407

1.052

$0.000,0.000$

$0.001,0.000$

$\mathrm{R} 1=2.7, \mathrm{wR} 2=7.7$

$\mathrm{R} 1=3.3, \mathrm{wR} 2=8.8$

$\mathrm{R} 1=2.7, \mathrm{wR} 2=7.7$

$\mathrm{R} 1=3.7, \mathrm{wR} 2=9.0$

$0.109,-0.106$

$0.132,-0.116$

627540 
Table 2. Fractional atomic coordinates (x 10^4) and equivalent isotropic displacement parameters $\left(A^{\wedge} 2 \times 10^{\wedge} 3\right)$ for 9

\begin{tabular}{|c|c|c|c|c|}
\hline Atom & $\mathrm{x}$ & $\mathrm{Y}$ & z & $\mathrm{U}(\mathrm{eq})$ \\
\hline$C(1)$ & $10396(3)$ & $6154(3)$ & $2384(2)$ & $61(1)$ \\
\hline$C(2)$ & $10817(3)$ & $6351(2)$ & $3169(2)$ & $55(1)$ \\
\hline$C(3)$ & $10984(3)$ & $5400(2)$ & $3648(2)$ & $63(1)$ \\
\hline$C(5)$ & $9958(4)$ & $4214(3)$ & $4367(2)$ & $65(1)$ \\
\hline$C(7)$ & $10081(3)$ & $7199(2)$ & $3542(2)$ & $52(1)$ \\
\hline$C(8)$ & $9438(3)$ & $7925(3)$ & $3002(2)$ & $69(1)$ \\
\hline C (9) & $9344(4)$ & $7468(3)$ & $2235(2)$ & $70(1)$ \\
\hline$C(10)$ & $9064(3)$ & $6286(3)$ & $2250(2)$ & $62(1)$ \\
\hline$C(11)$ & $10556(4)$ & $7185(3)$ & $1967(2)$ & $81(1)$ \\
\hline$C(12)$ & $8159(3)$ & $5887(3)$ & $2774(2)$ & 71 (1) \\
\hline$C(13)$ & $8739(4)$ & $5883(3)$ & $1483(2)$ & $90(1)$ \\
\hline$C(14)$ & $10620(3)$ & $9378(3)$ & $5185(2)$ & $60(1)$ \\
\hline$C(15)$ & $10084(3)$ & $10386(2)$ & $5382(2)$ & $63(1)$ \\
\hline$C(16)$ & $10796(5)$ & $11123(3)$ & $5697(2)$ & $88(1)$ \\
\hline C (17) & $10347(7)$ & $12106(4)$ & $5870(3)$ & $120(2)$ \\
\hline C (18) & $9226(7)$ & $12318(4)$ & $5736(3)$ & $122(2)$ \\
\hline C (19) & $8513(5)$ & $11588(4)$ & $5437(3)$ & $116(2)$ \\
\hline$C(20)$ & $8941(4)$ & $10630(3)$ & $5256(2)$ & $84(1)$ \\
\hline$C(21)$ & $9475(3)$ & $5426(3)$ & $6587(2)$ & $64(1)$ \\
\hline$C(22)$ & $9114(3)$ & $5954(2)$ & $5871(2)$ & $52(1)$ \\
\hline$C(23)$ & $8981(3)$ & $5255(2)$ & $5212(2)$ & $55(1)$ \\
\hline C (25) & $9979(2)$ & $8620(2)$ & $4697(2)$ & $54(1)$ \\
\hline C (27) & $9875(3)$ & $6912(2)$ & $5720(2)$ & $54(1)$ \\
\hline$C(28)$ & $10486(4)$ & $7351(3)$ & $6413(2)$ & $77(1)$ \\
\hline C (29) & $10511(4)$ & $6589(3)$ & $7044(2)$ & $79(1)$ \\
\hline$C(30)$ & $10781(3)$ & $5468(3)$ & $6783(2)$ & $70(1)$ \\
\hline$C(31)$ & $9279(4)$ & $6225(4)$ & $7202(2)$ & $93(1)$ \\
\hline C (32) & $11726(3)$ & $5304(3)$ & $6216(2)$ & $76(1)$ \\
\hline C (33) & $11033(5)$ & $4726(4)$ & $7425(2)$ & $108(2)$ \\
\hline $\mathrm{O}(4)$ & $9933(2)$ & $4881(2)$ & $3765(1)$ & $60(1)$ \\
\hline$O(14)$ & $11584(2)$ & $9152(2)$ & $5404(2)$ & $83(1)$ \\
\hline O (24) & $10026(2)$ & $4737(2)$ & $5040(1)$ & $57(1)$ \\
\hline$S(6)$ & $11022(1)$ & $7888(1)$ & $4174(1)$ & $58(1)$ \\
\hline$S(26)$ & $8966(1)$ & $7857(1)$ & $5244(1)$ & $59(1)$ \\
\hline
\end{tabular}


S15

Table 3. Fractional atomic coordinates $\left(x 10^{\wedge} 4\right)$ and equivalent isotropic displacement parameters $\left(A^{\wedge} 2 \times 10^{\wedge} 3\right)$ for $12 j$

\begin{tabular}{|c|c|c|c|c|}
\hline Atom & $\mathrm{x}$ & $\mathrm{Y}$ & z & $\mathrm{U}(\mathrm{eq})$ \\
\hline$C(1)$ & $6804(6)$ & $10529(3)$ & $9836(1)$ & $65(1)$ \\
\hline C (2) & $6968(5)$ & $10211(3)$ & $9433(1)$ & $63(1)$ \\
\hline$C(3)$ & $6796(7)$ & $11218(3)$ & $9168(1)$ & $68(1)$ \\
\hline C (5) & $4441(9)$ & $12563(3)$ & $8926(1)$ & $78(1)$ \\
\hline C (7) & $5460(6)$ & $9218(3)$ & $9343(1)$ & $59(1)$ \\
\hline$C(8)$ & $4819(7)$ & $8490(4)$ & $9676(1)$ & $74(1)$ \\
\hline$C(9)$ & $5201(7)$ & $9098(3)$ & $10036(1)$ & $72(1)$ \\
\hline$C(10)$ & $4641(6)$ & $10404(3)$ & $10015(1)$ & $64(1)$ \\
\hline$C(11)$ & $7450(8)$ & $9446(4)$ & $10051(1)$ & $83(1)$ \\
\hline$C(12)$ & $2651(6)$ & $10752(4)$ & $9834(1)$ & $77(1)$ \\
\hline$C(13)$ & $4652(8)$ & $10983(4)$ & 10390 (1) & $91(1)$ \\
\hline$C(14)$ & $3831(5)$ & $6508(3)$ & $8948(1)$ & $61(1)$ \\
\hline$C(15)$ & $2633(6)$ & $5742(2)$ & $8697(1)$ & $59(1)$ \\
\hline$C(16)$ & $601(7)$ & $5517(3)$ & $8766(1)$ & $76(1)$ \\
\hline$C(17)$ & $-539(9)$ & $4808(4)$ & $8542(1)$ & $98(1)$ \\
\hline C (18) & $377(10)$ & $4310(4)$ & $8246(2)$ & $105(2)$ \\
\hline C (19) & $2411(10)$ & $4539(4)$ & $8169(1)$ & $96(2)$ \\
\hline$C(20)$ & $3560(7)$ & $5251(3)$ & $8396(1)$ & $76(1)$ \\
\hline$C(21)$ & $1084(6)$ & $11004(3)$ & $7980(1)$ & $63(1)$ \\
\hline C (22) & $1450(6)$ & $10543(3)$ & $8364(1)$ & $58(1)$ \\
\hline C (23) & $1866(6)$ & $11425(3)$ & $8658(1)$ & $64(1)$ \\
\hline C (25) & $4475(5)$ & $7643(3)$ & $8756(1)$ & $59(1)$ \\
\hline$C(27)$ & $3047(6)$ & $9557(3)$ & $8361(1)$ & $58(1)$ \\
\hline$C(28)$ & $3493(8)$ & $9024(4)$ & $7983(1)$ & $75(1)$ \\
\hline$C(29)$ & $2594(7)$ & $9730(3)$ & $7674(1)$ & $75(1)$ \\
\hline$C(30)$ & $2999(6)$ & $11051(3)$ & $7724(1)$ & $66(1)$ \\
\hline$C(31)$ & $338(8)$ & $9969(4)$ & $7753(1)$ & $82(1)$ \\
\hline C (32) & $5095(7)$ & $11432(4)$ & 7851 (1) & $84(1)$ \\
\hline$C(33)$ & $2475(9)$ & $11739(3)$ & $7379(1)$ & $102(2)$ \\
\hline $\mathrm{O}(4)$ & $4944(4)$ & $11827(2)$ & $9219(1)$ & 71 (1) \\
\hline$O(14)$ & $5565(5)$ & $5887(2)$ & 9075 (1) & $82(1)$ \\
\hline$O(24)$ & $3821(4)$ & $11942(2)$ & $8616(1)$ & $68(1)$ \\
\hline$S(6)$ & $6615(1)$ & $8391(1)$ & $8976(1)$ & $69(1)$ \\
\hline$S(26)$ & $2183(1)$ & $8491(1)$ & $8691(1)$ & $59(1)$ \\
\hline
\end{tabular}




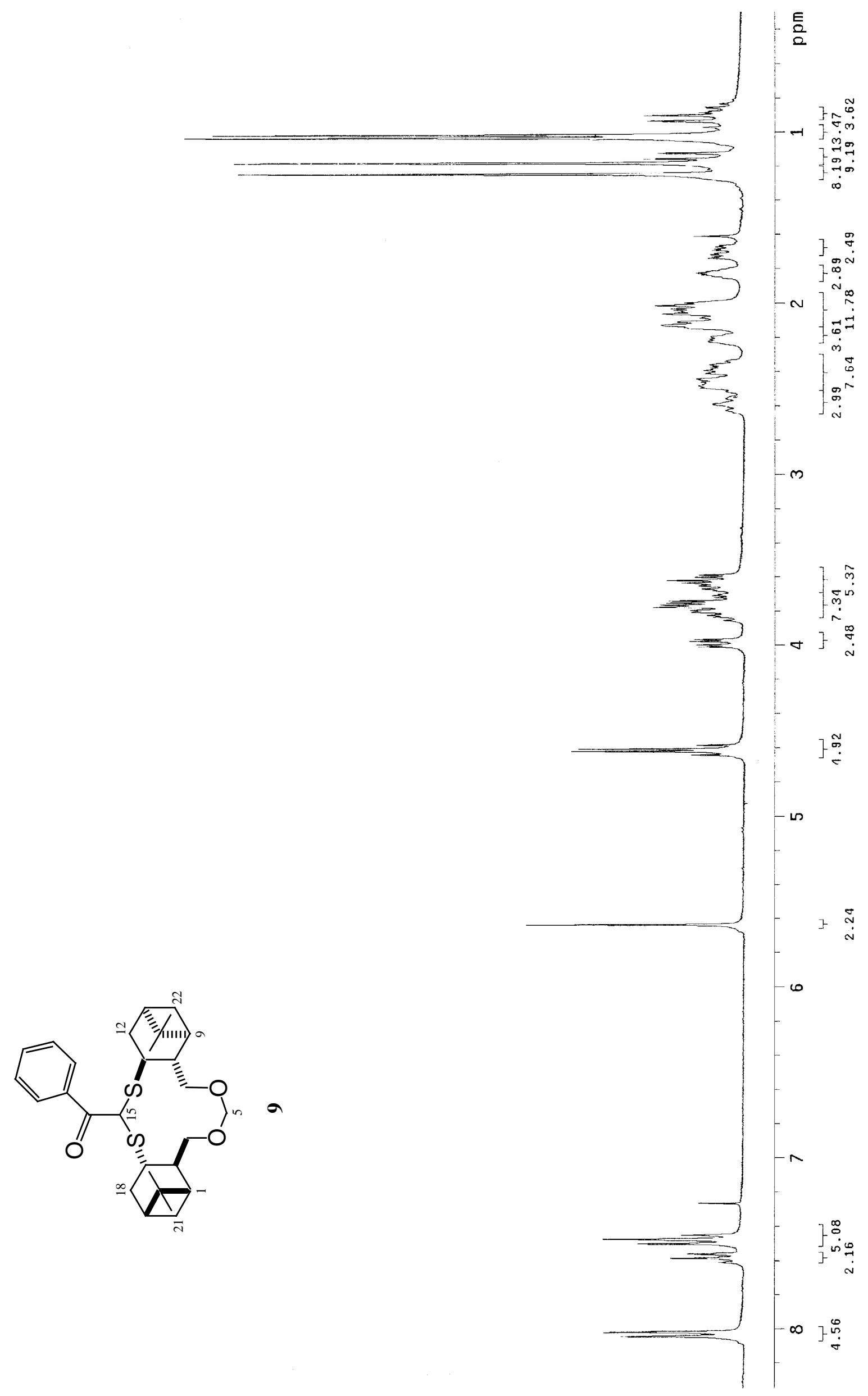




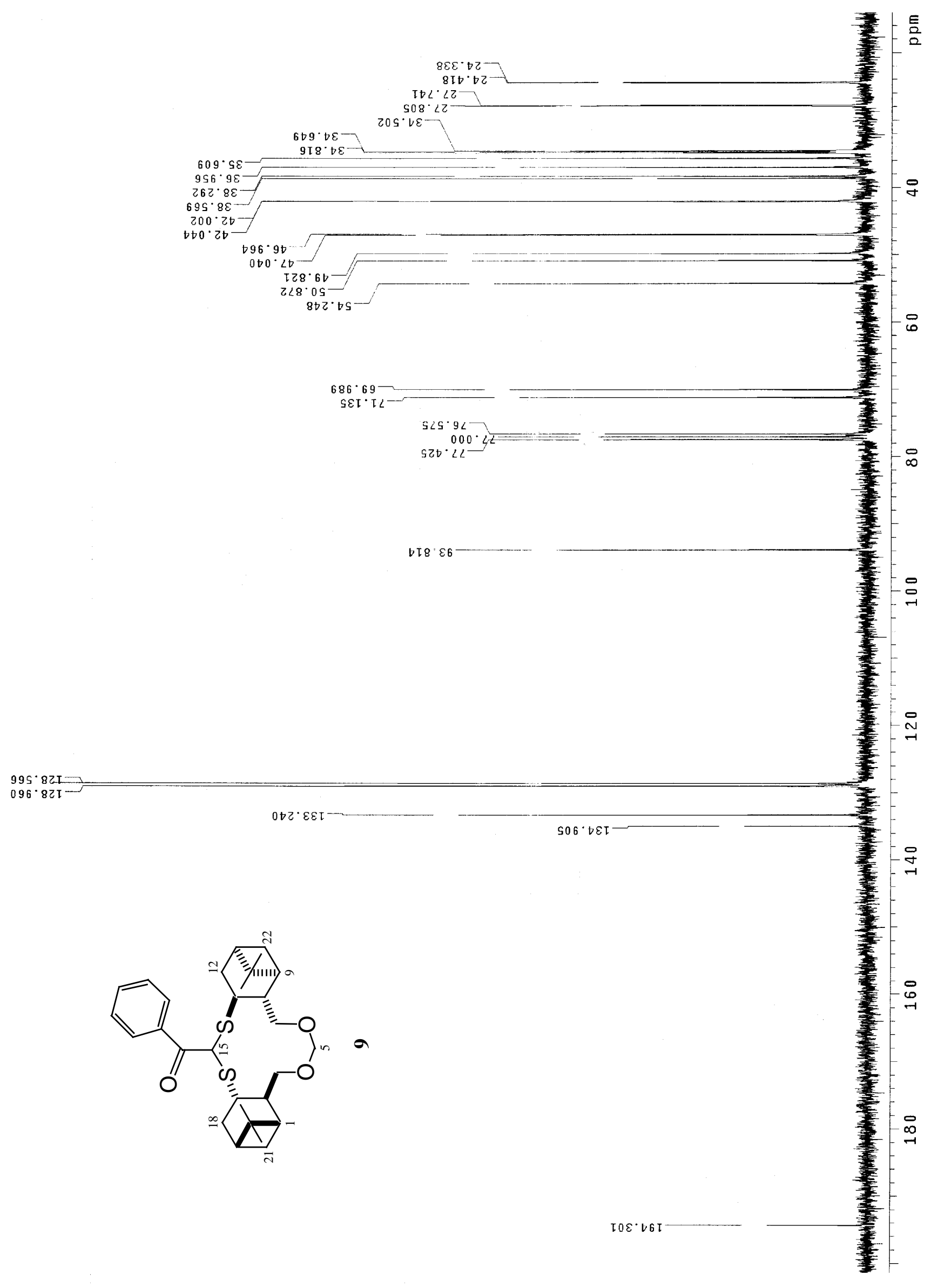




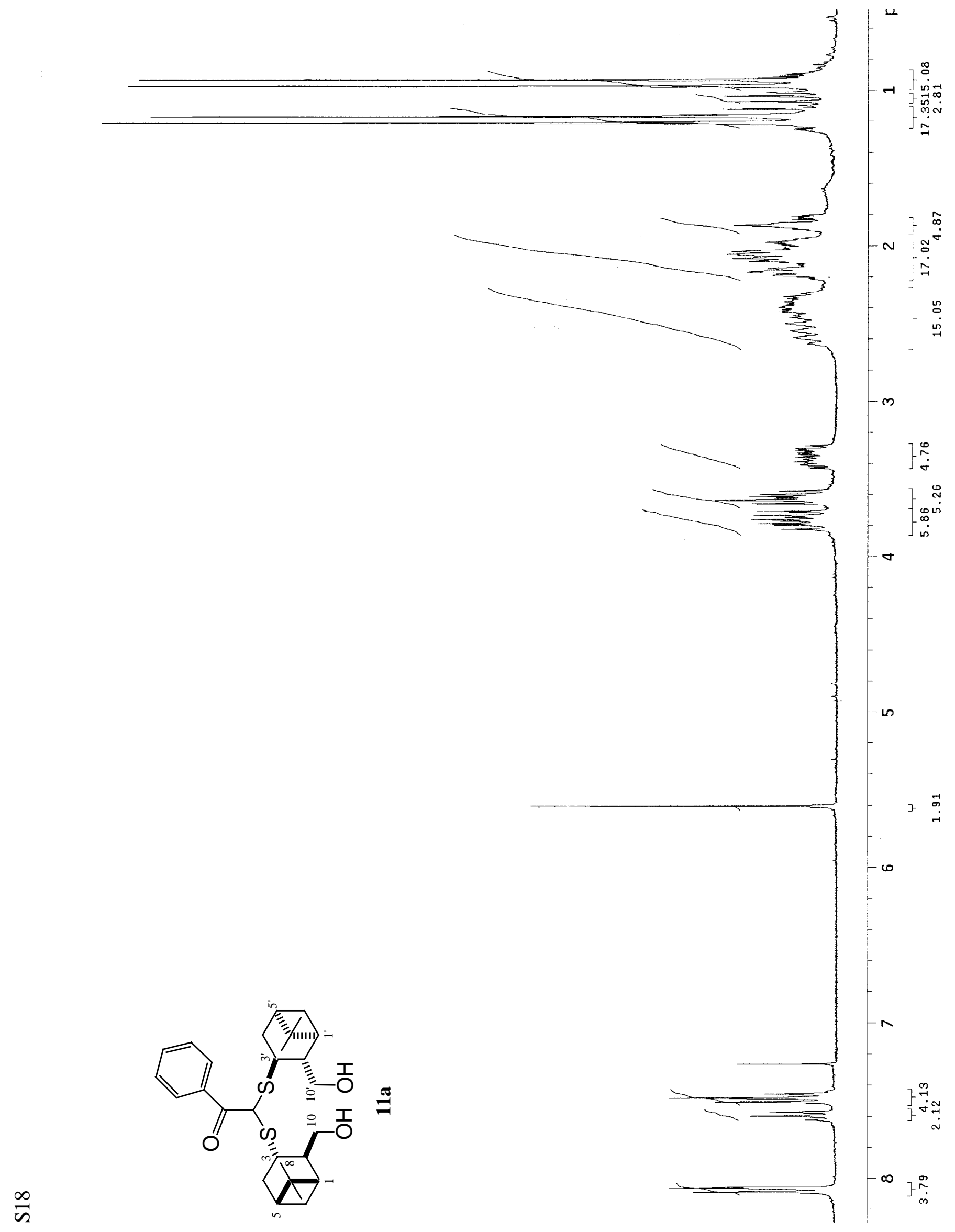




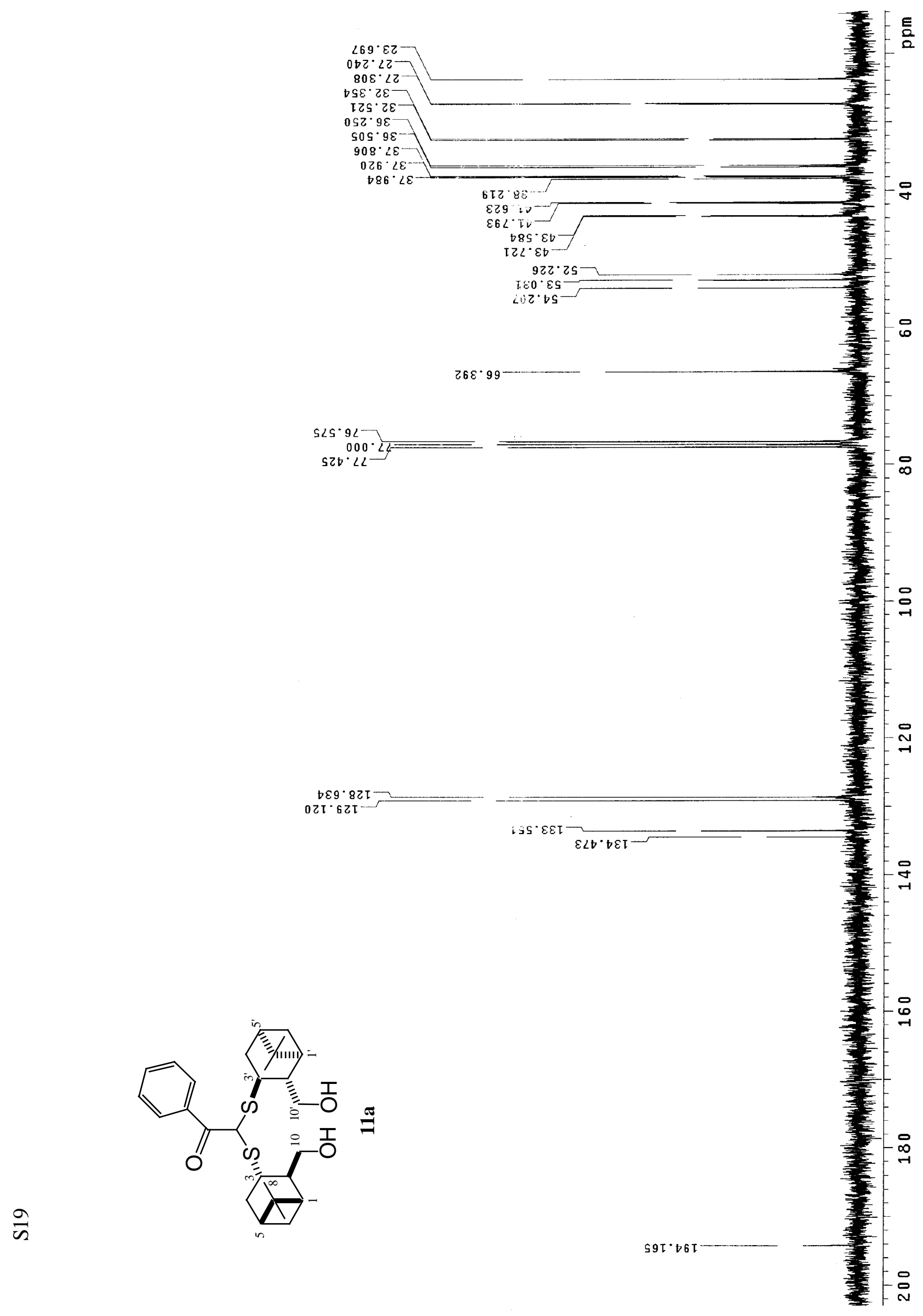




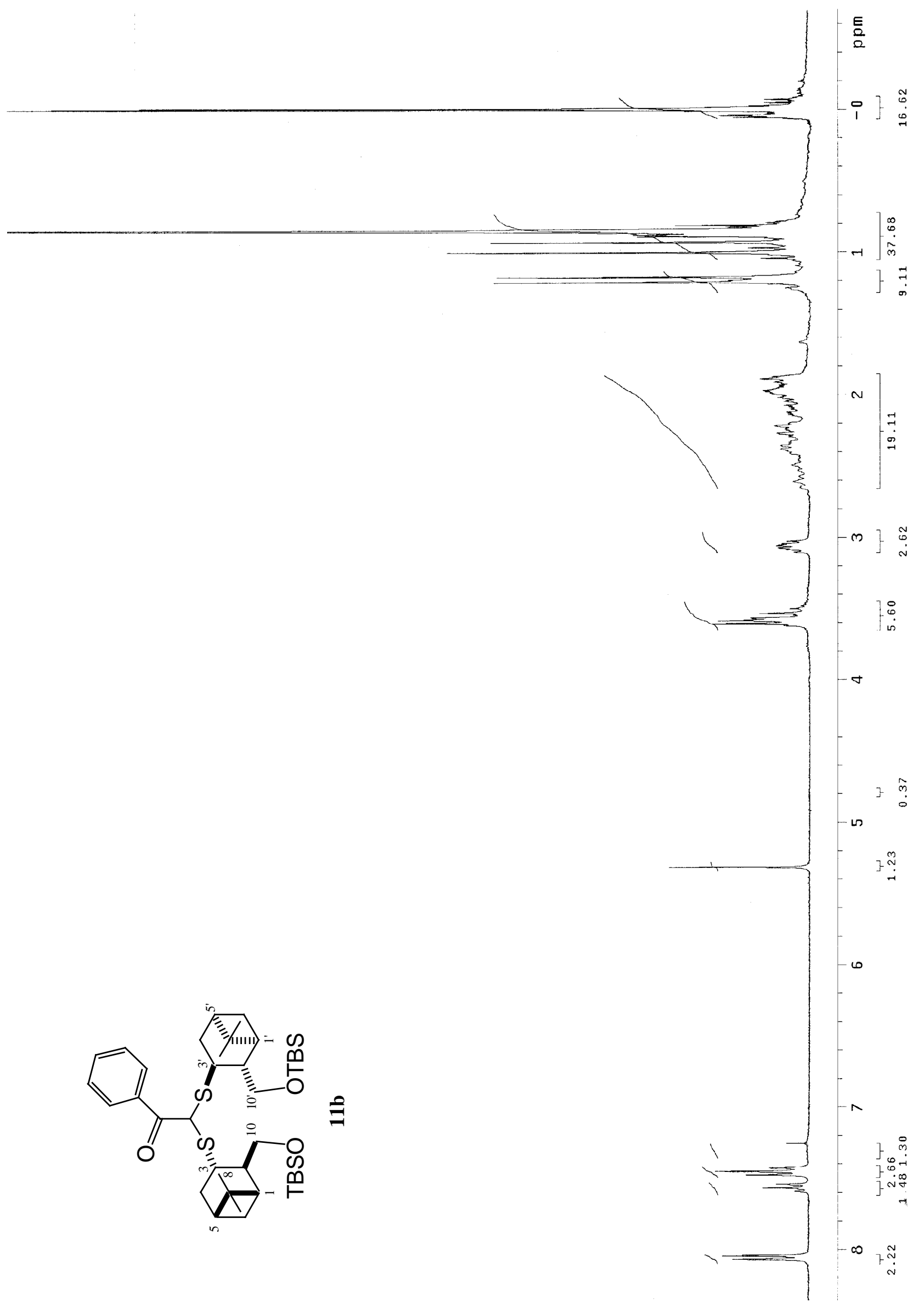




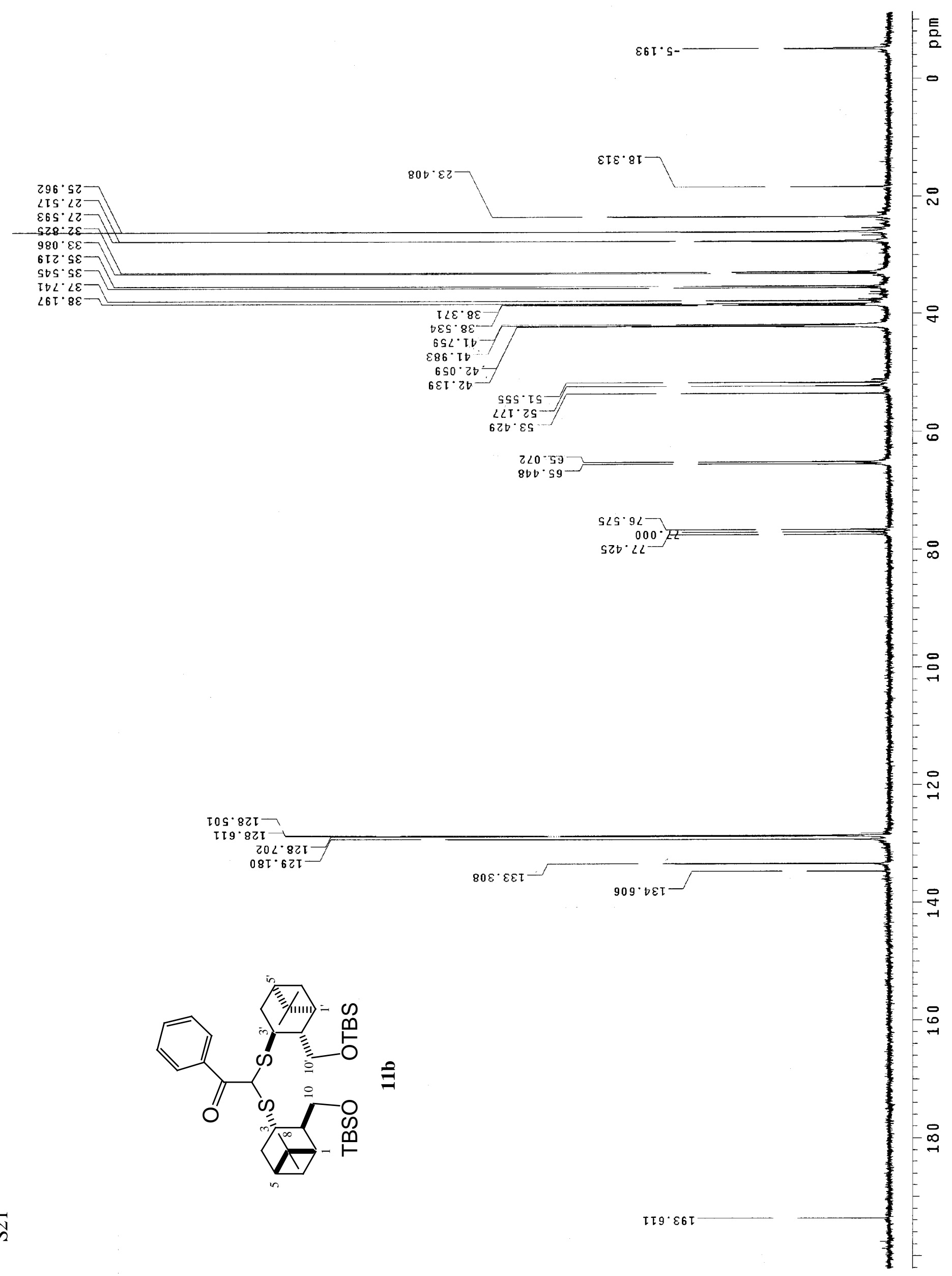




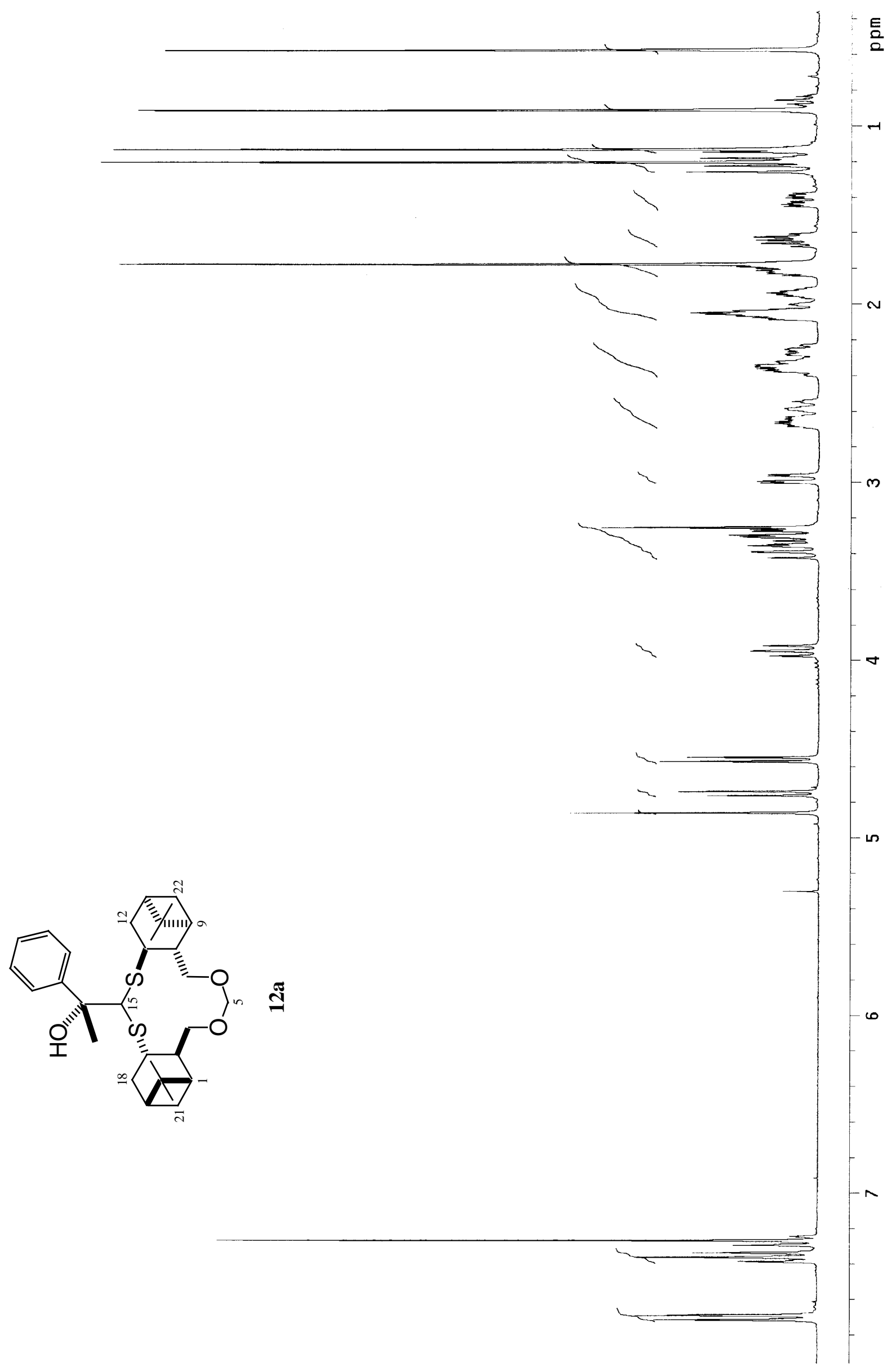




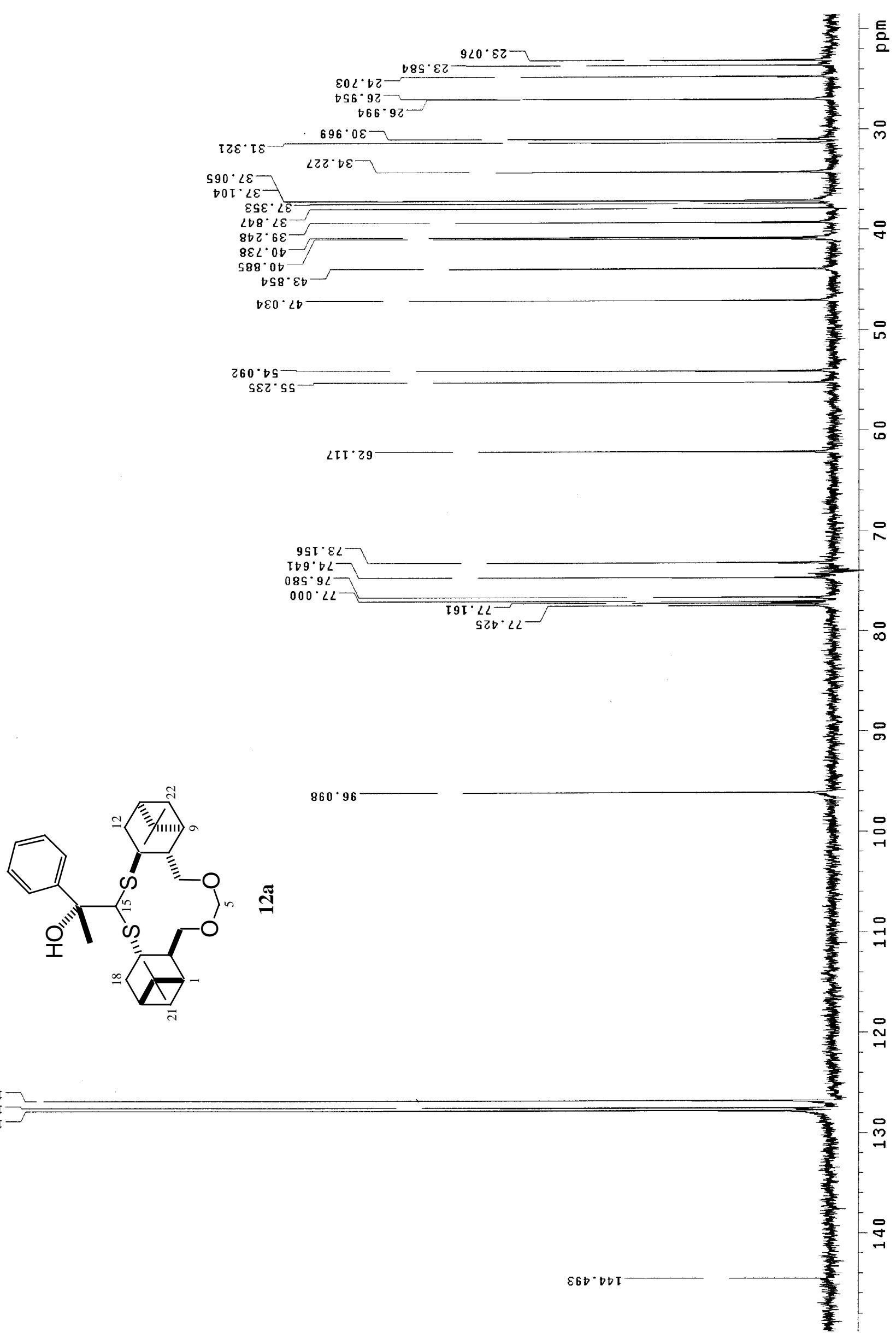




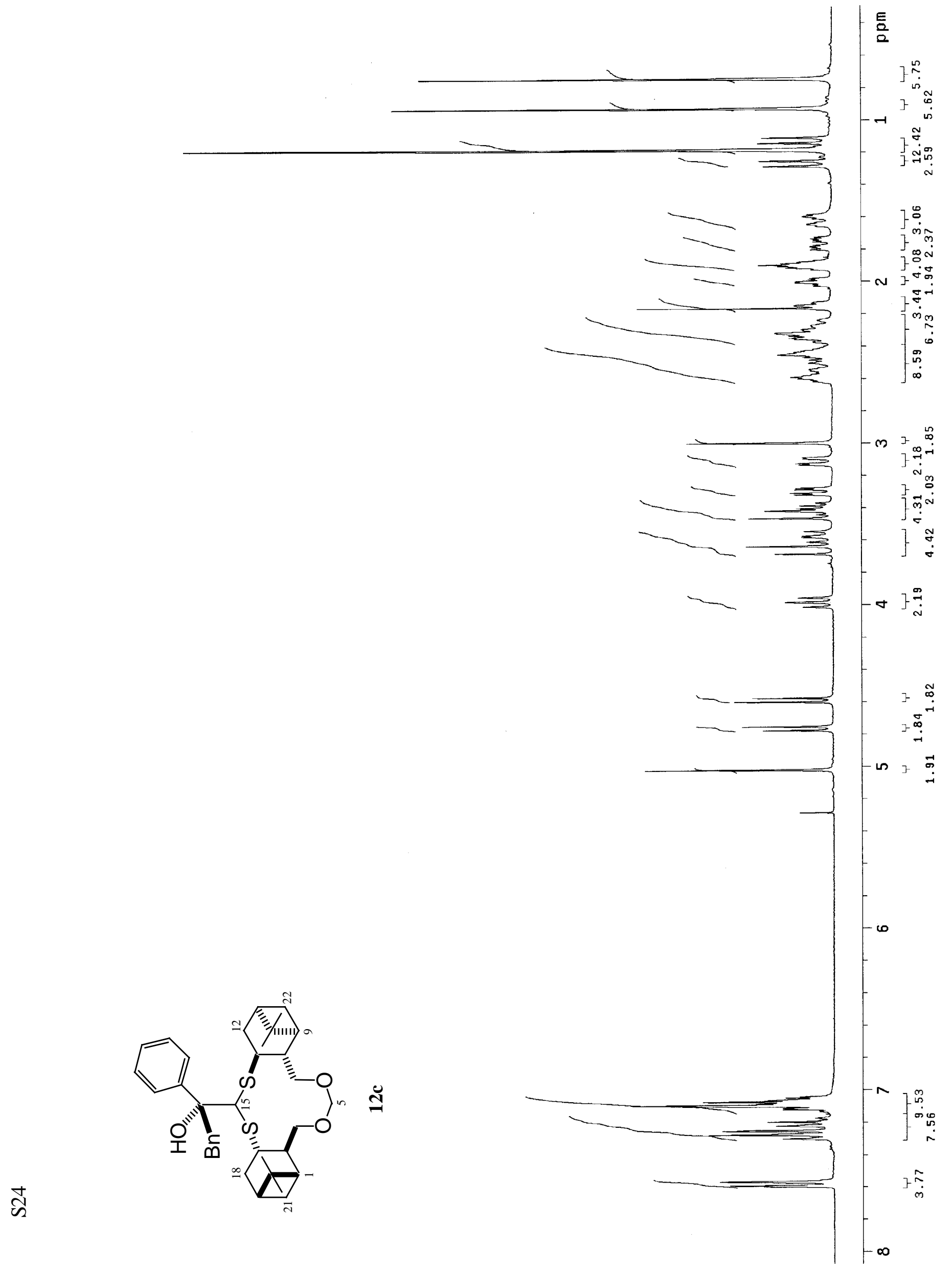




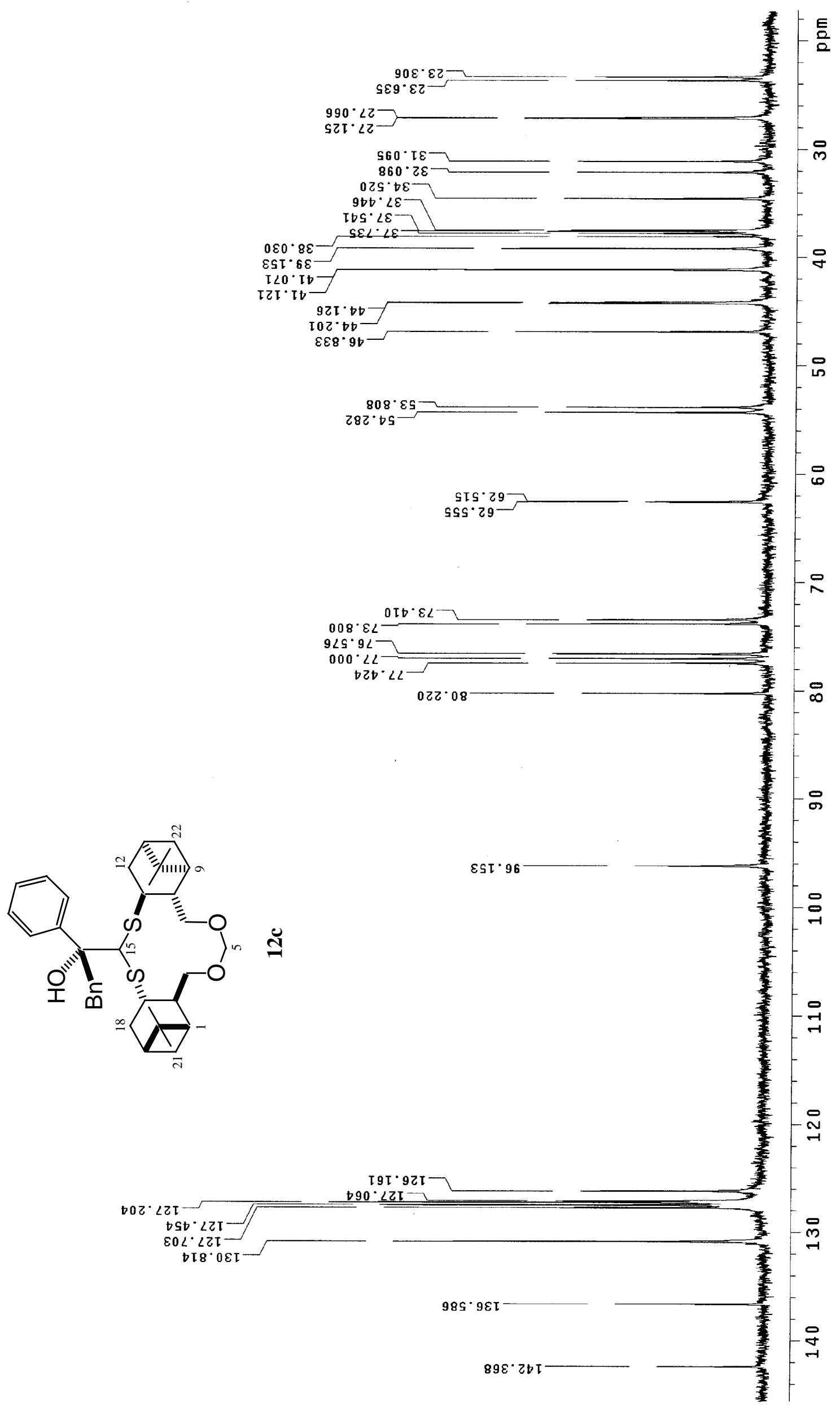




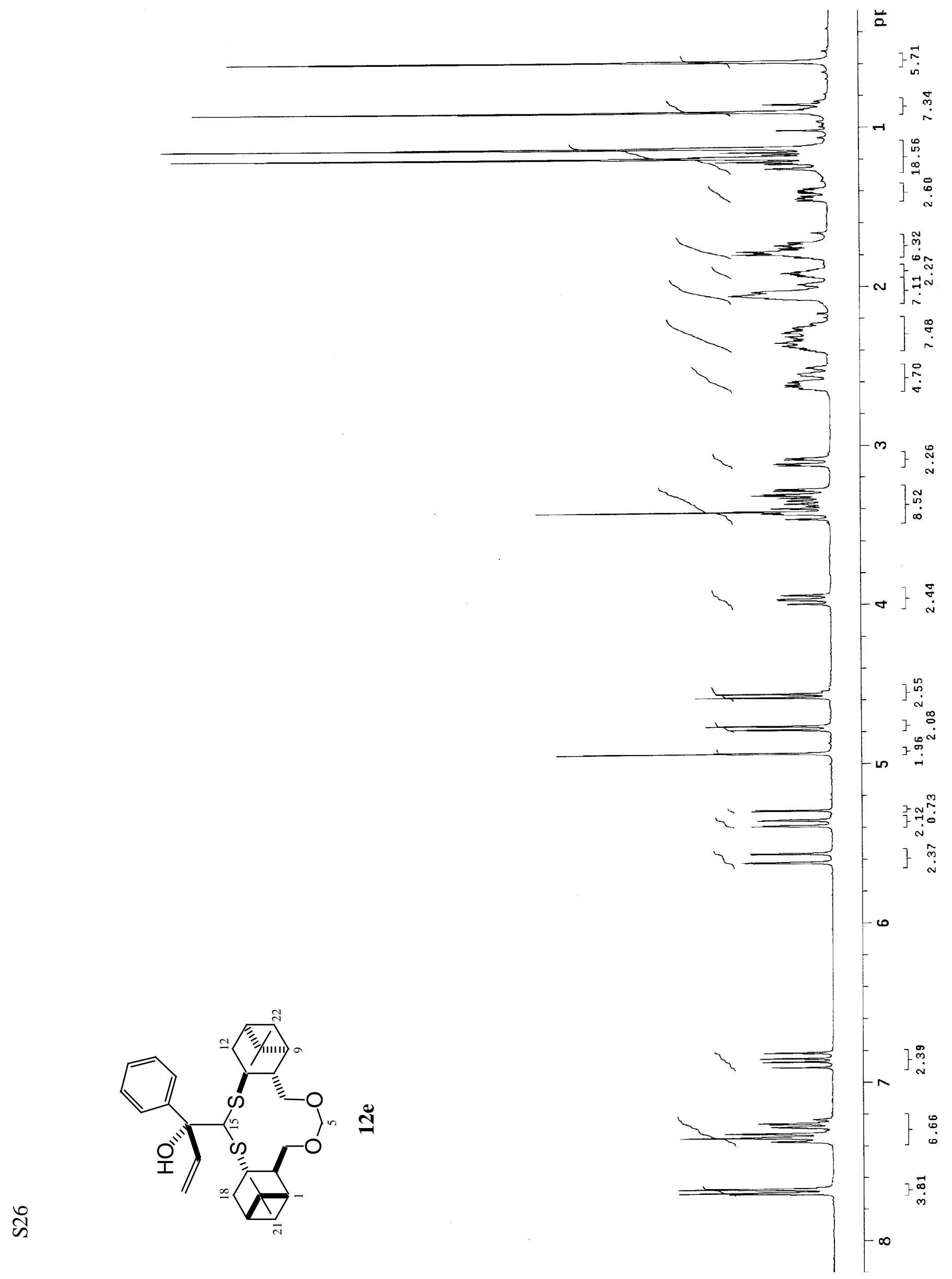




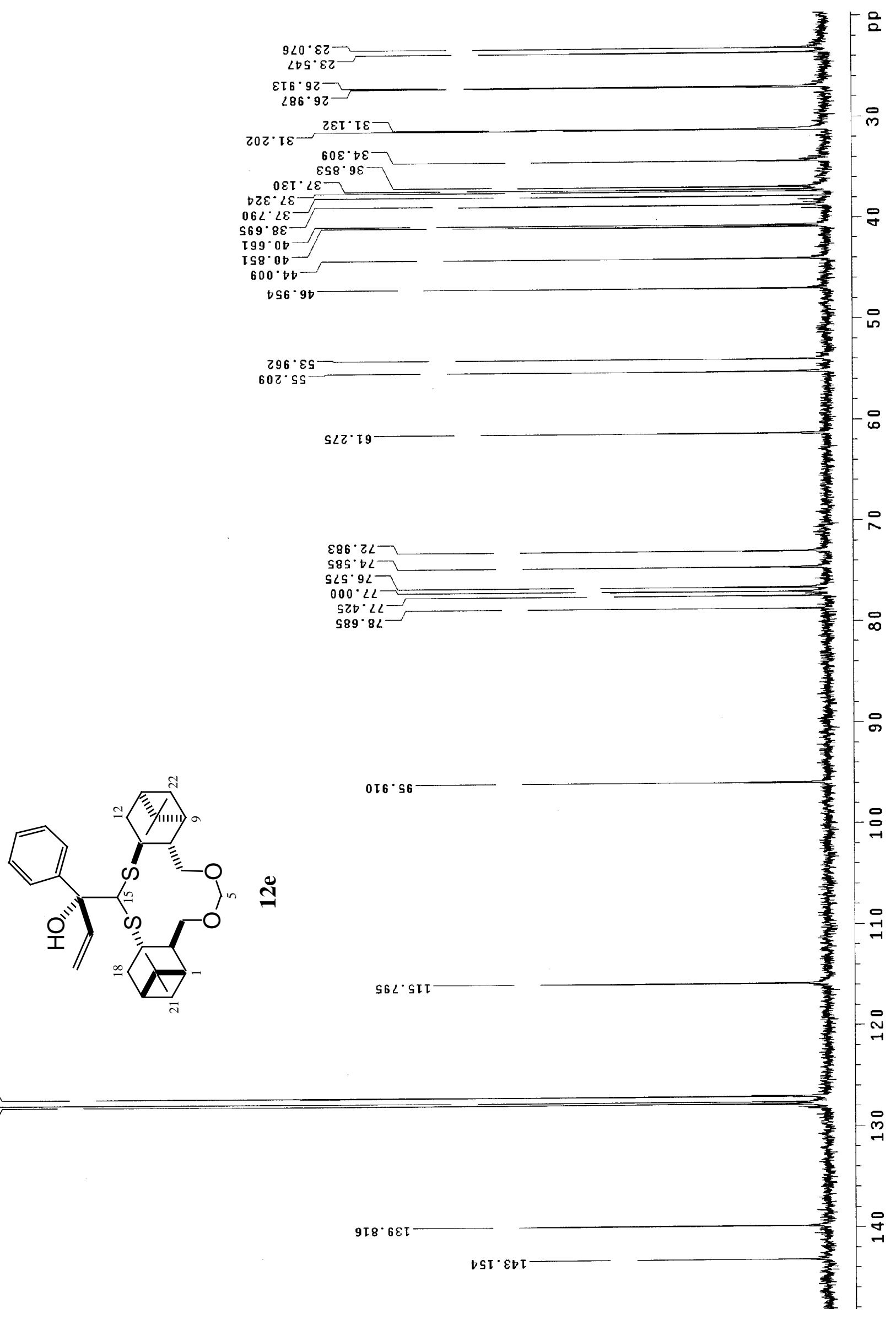




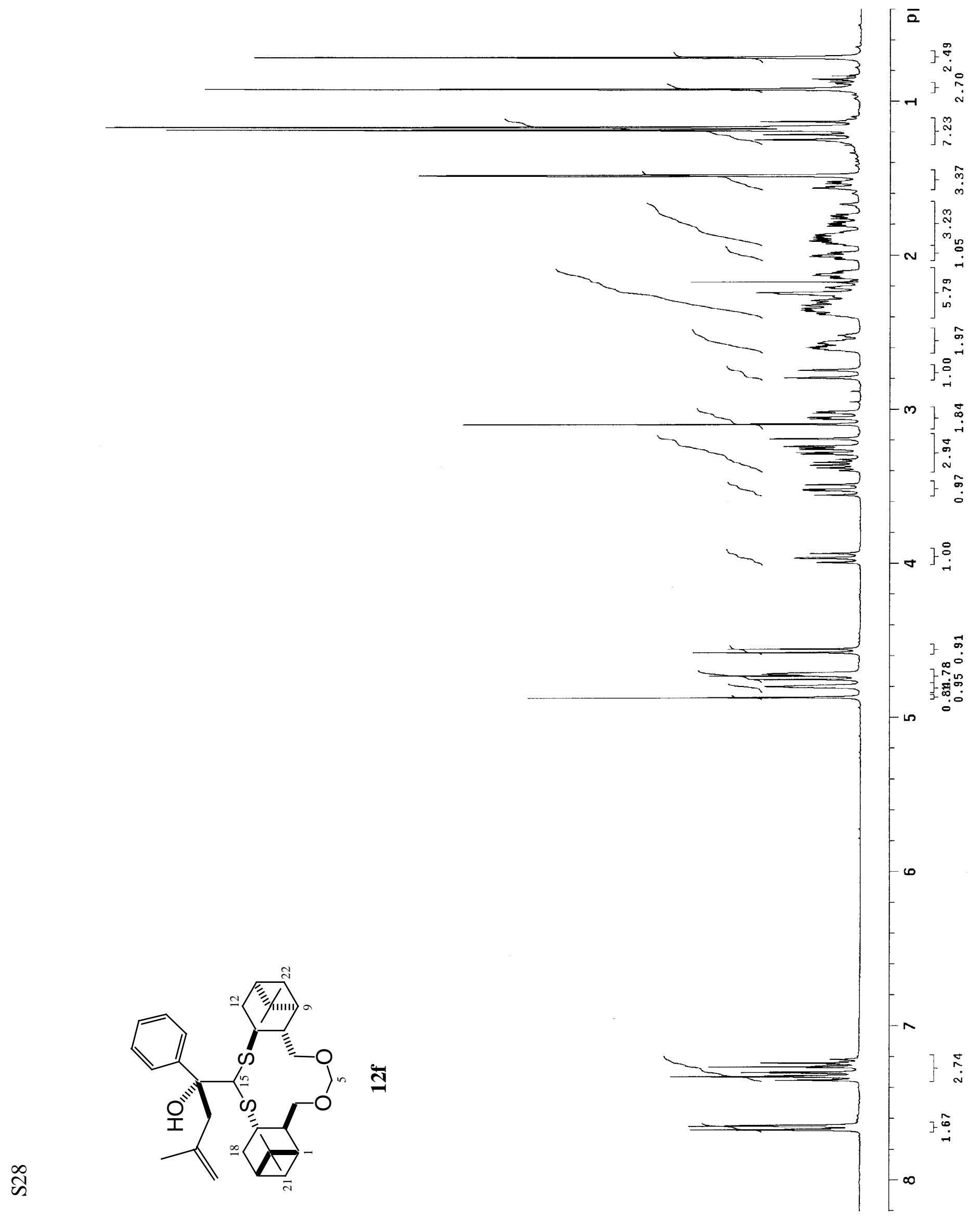




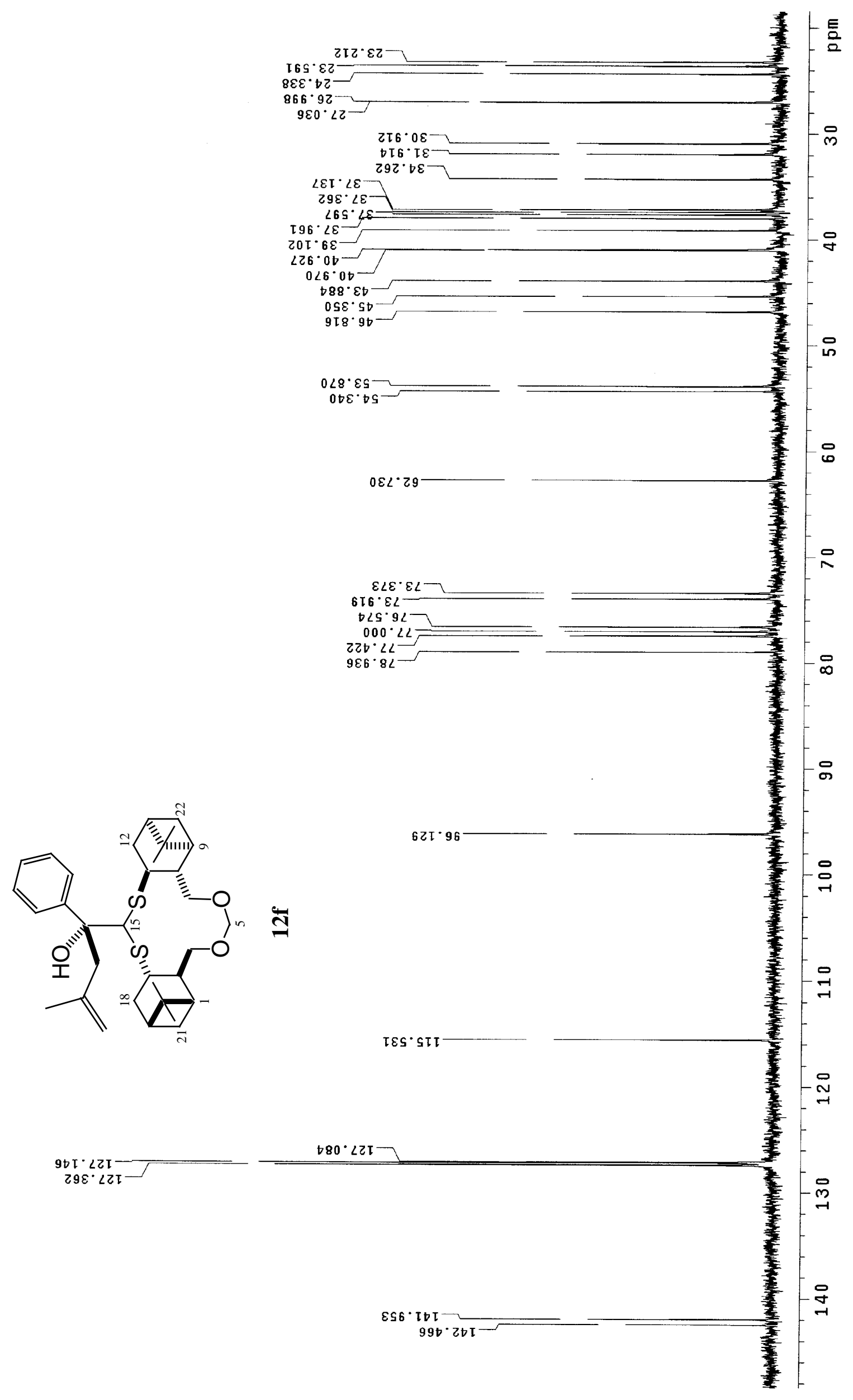




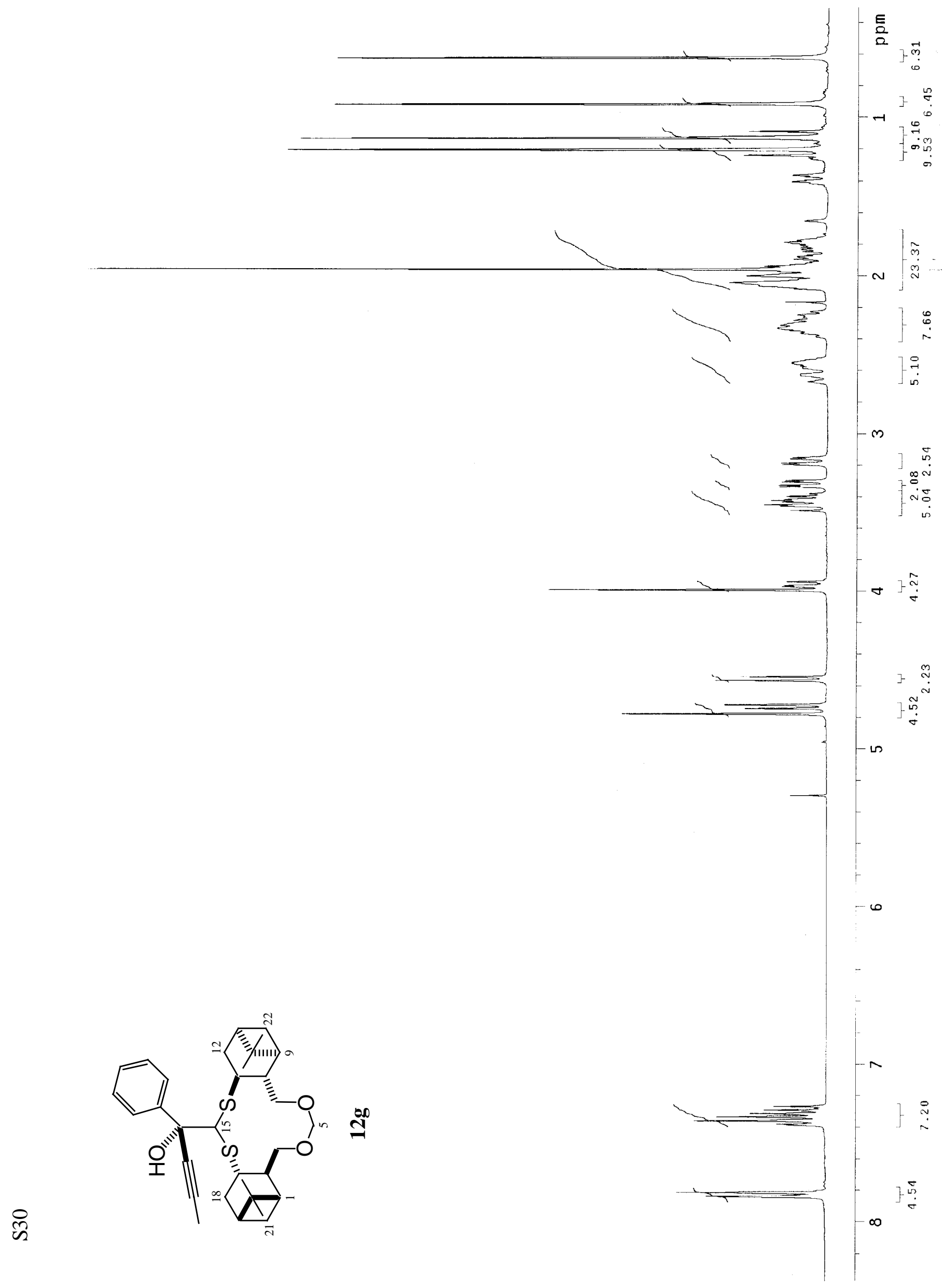




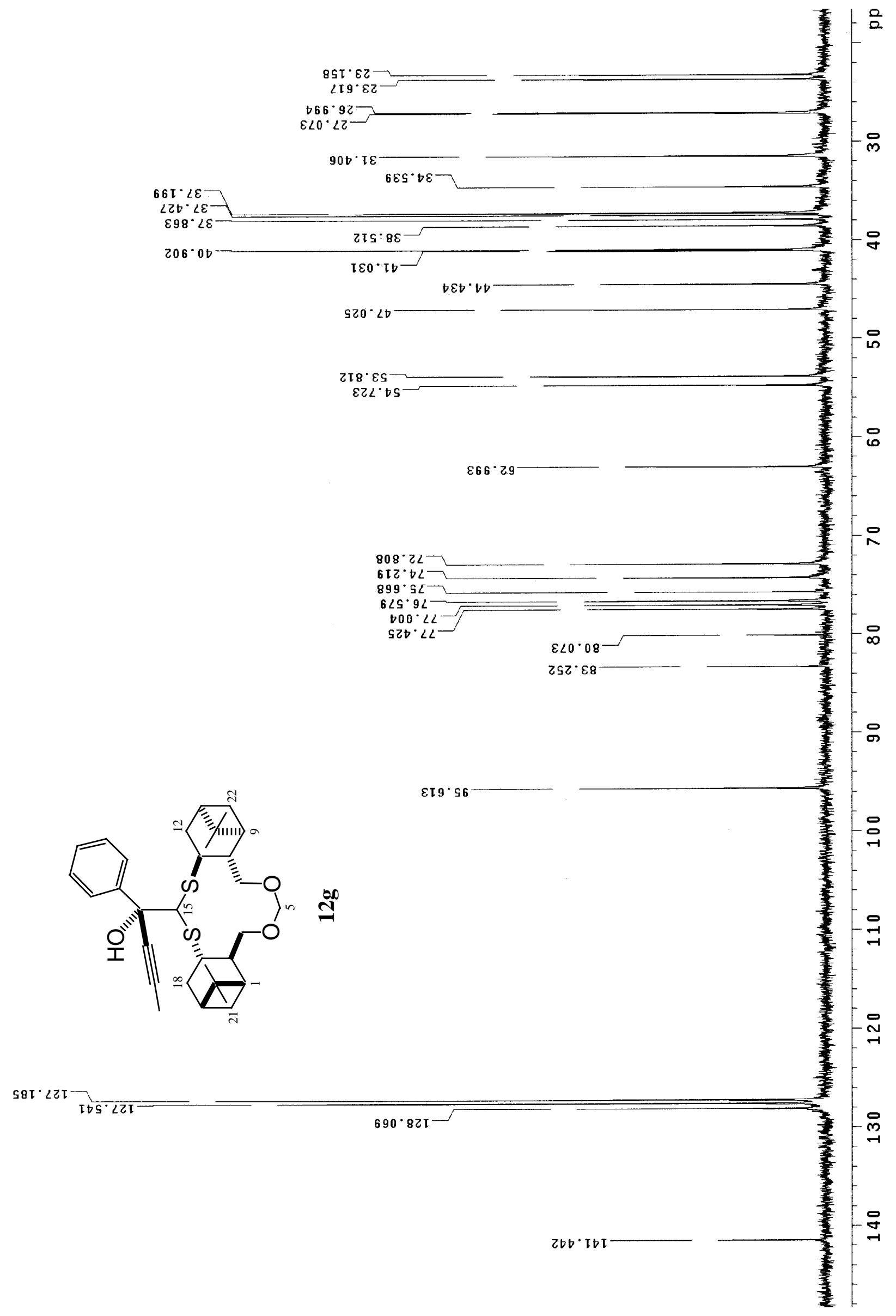




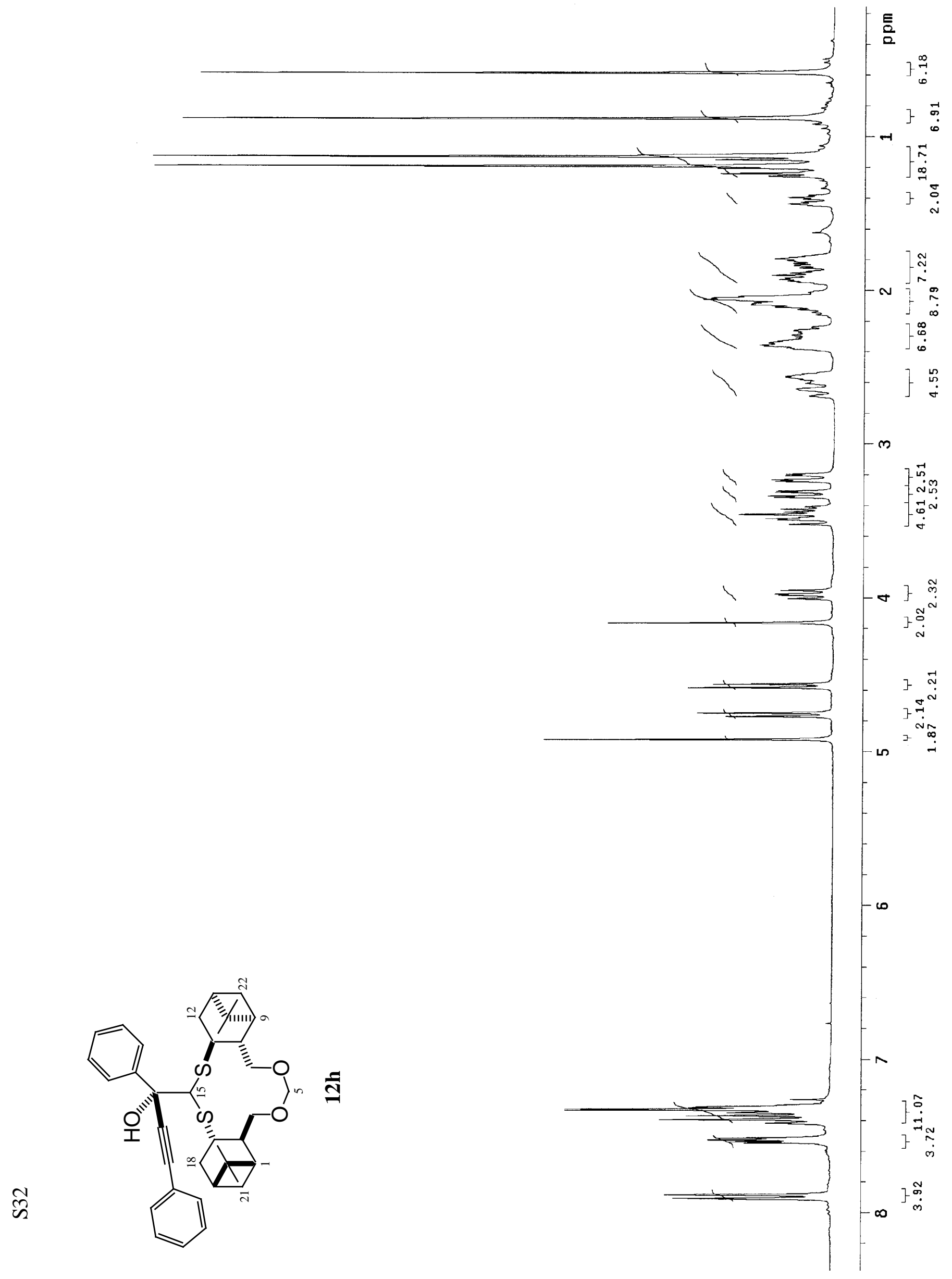




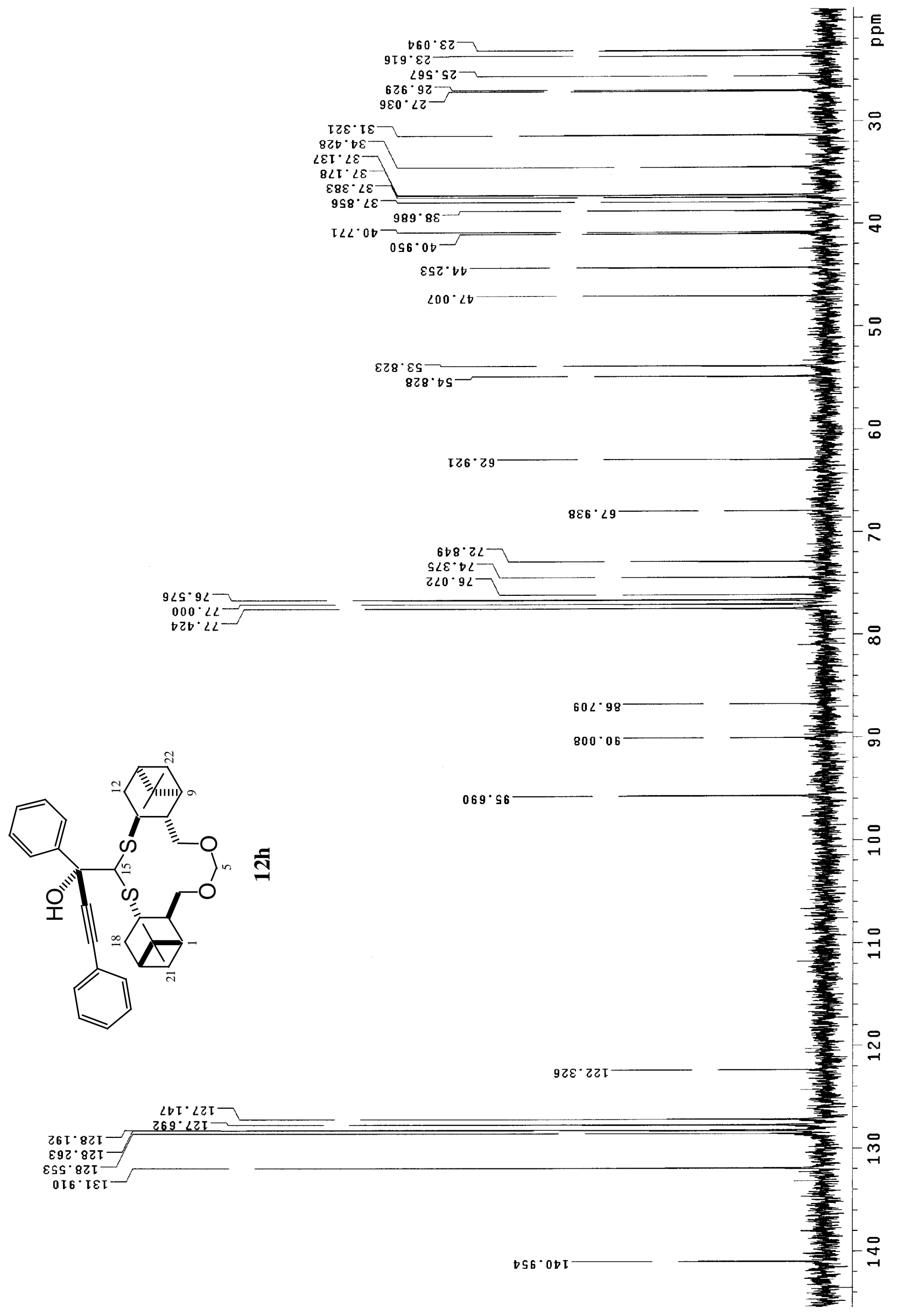




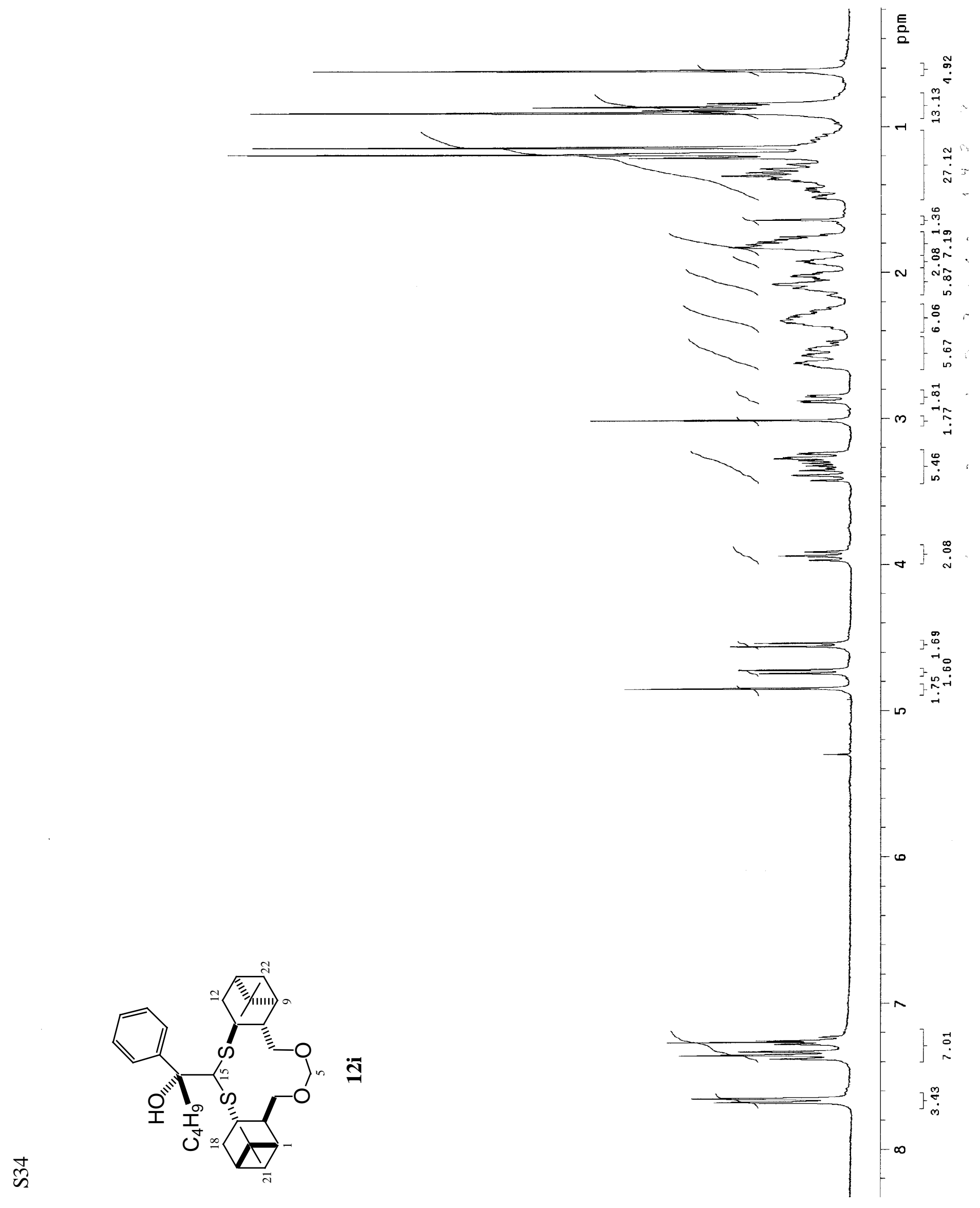




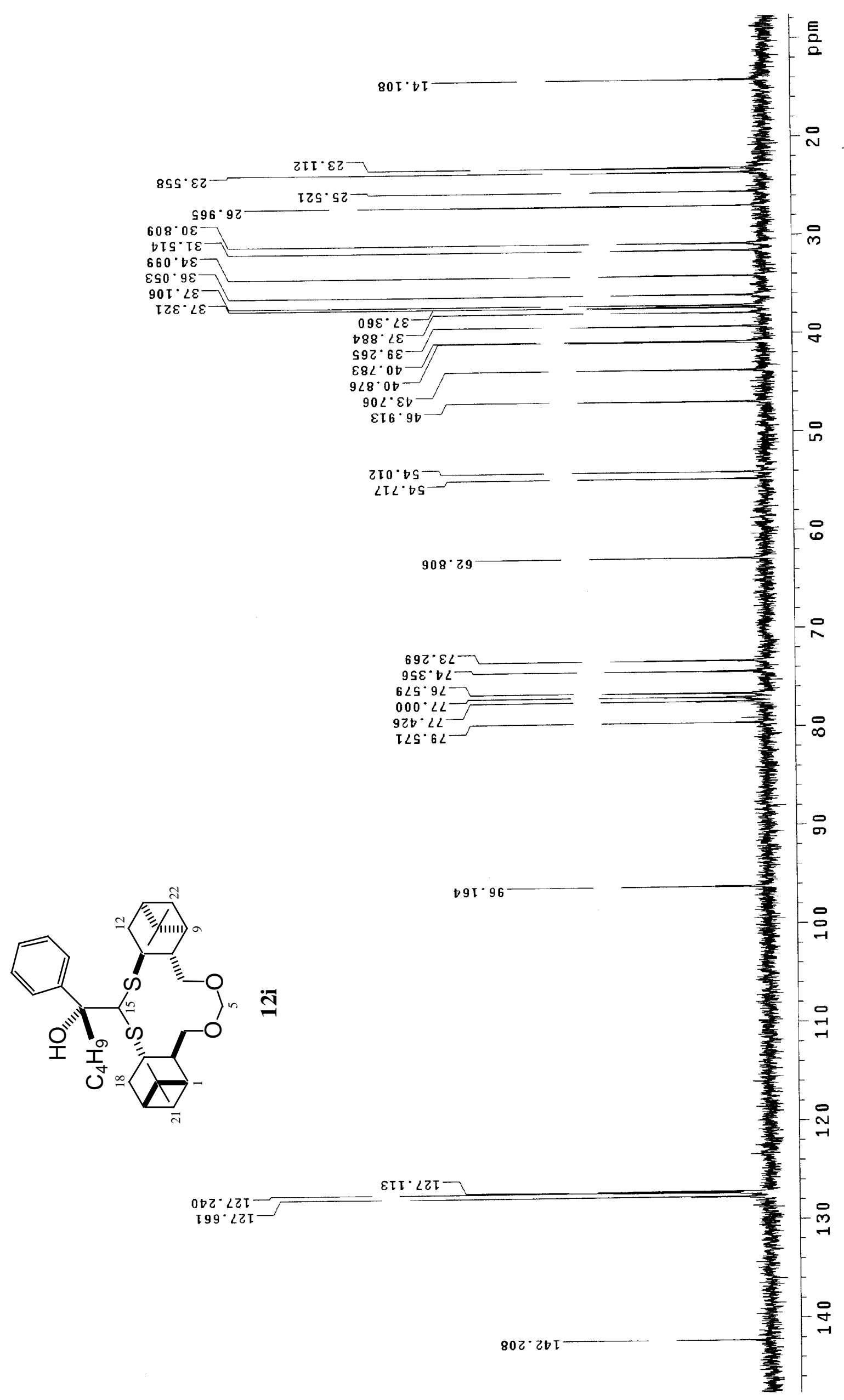




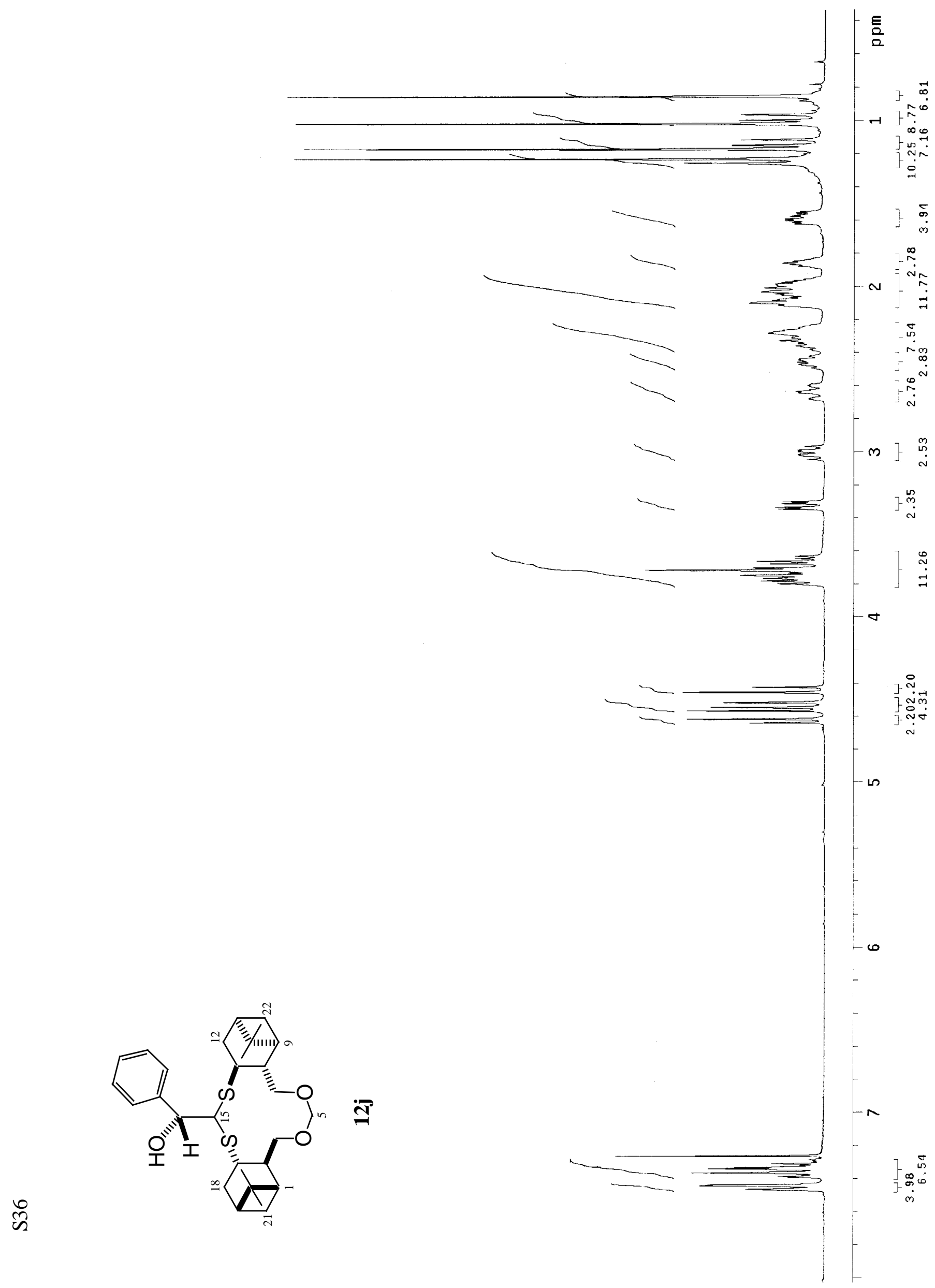




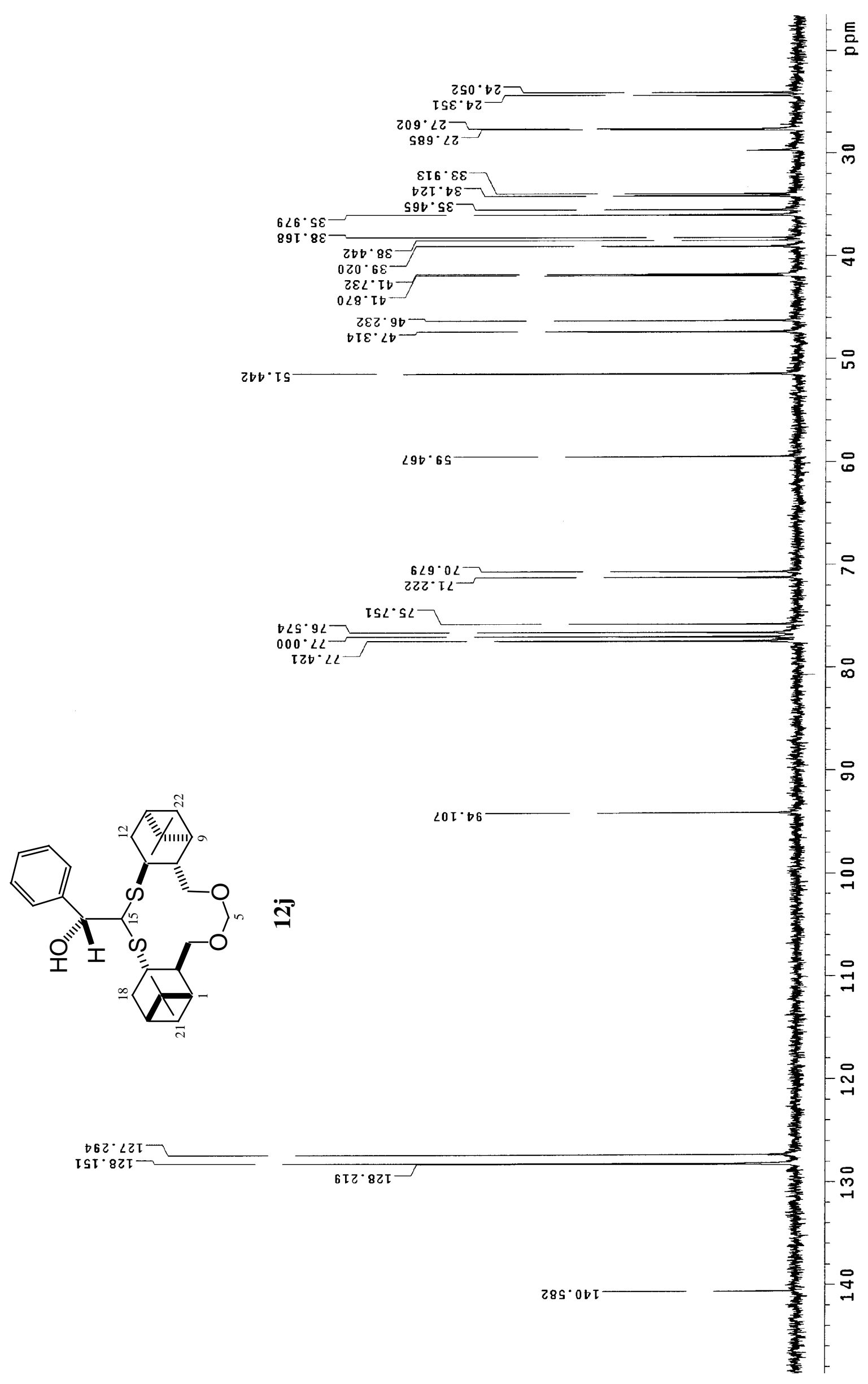




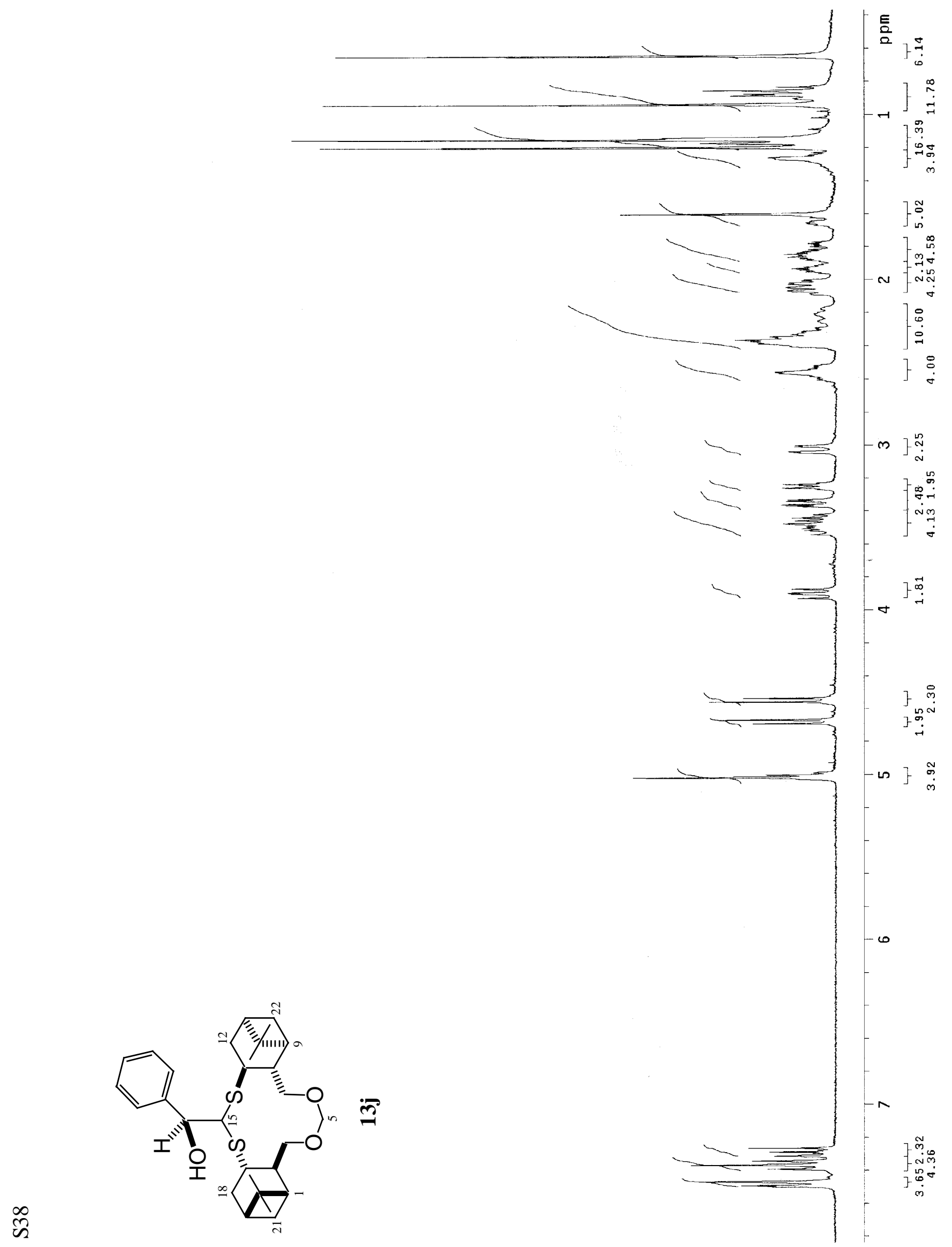




$$
\text { A }
$$




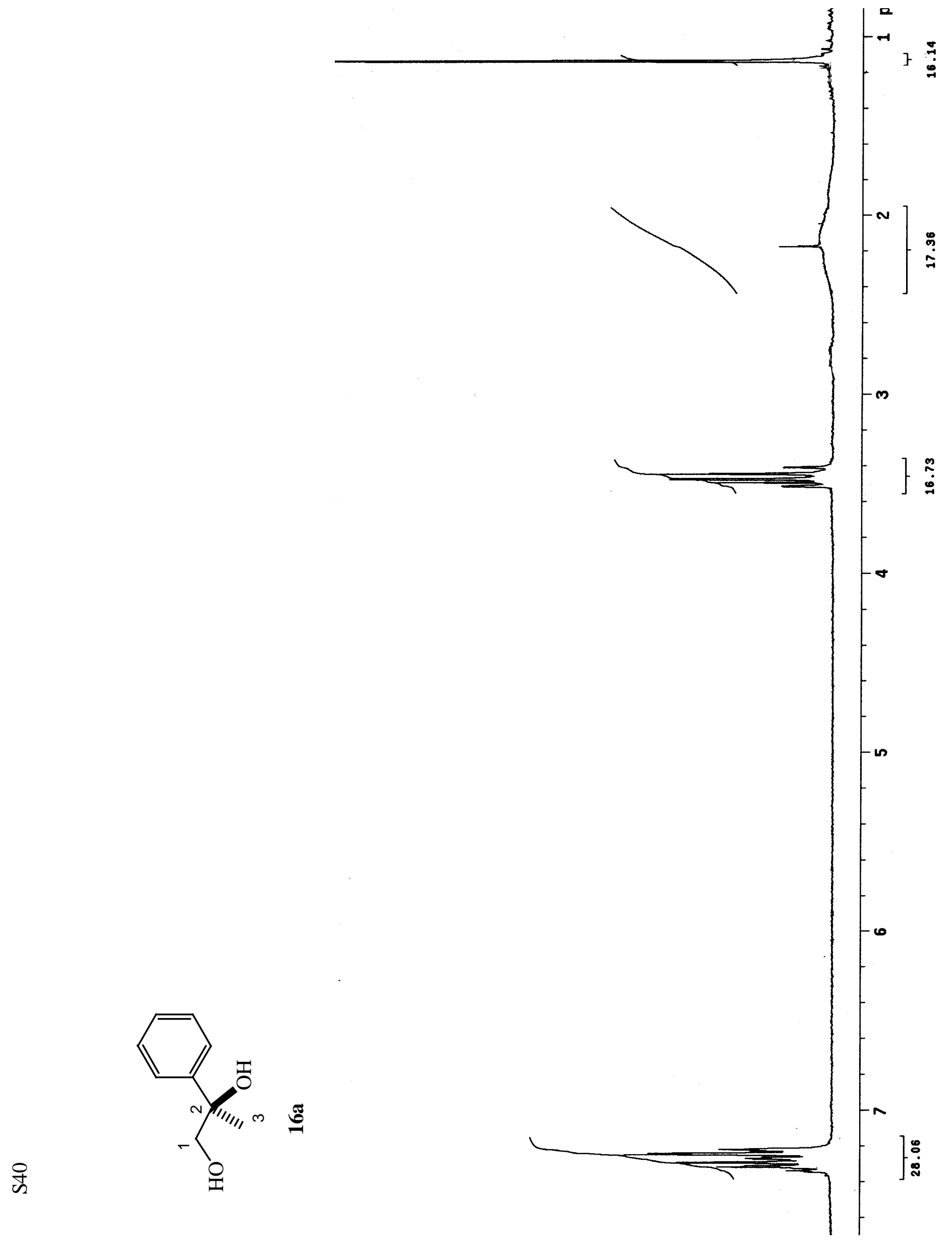




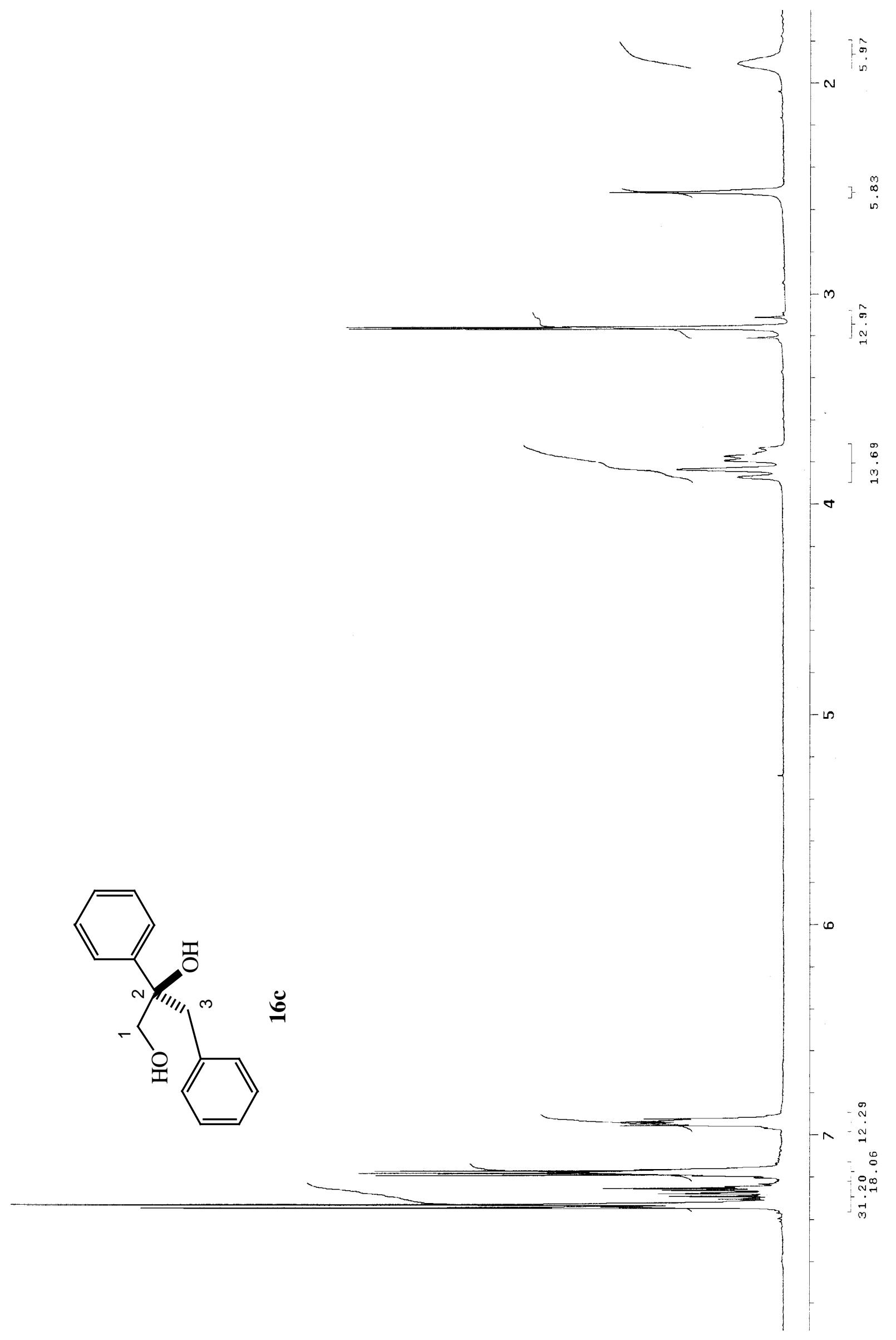




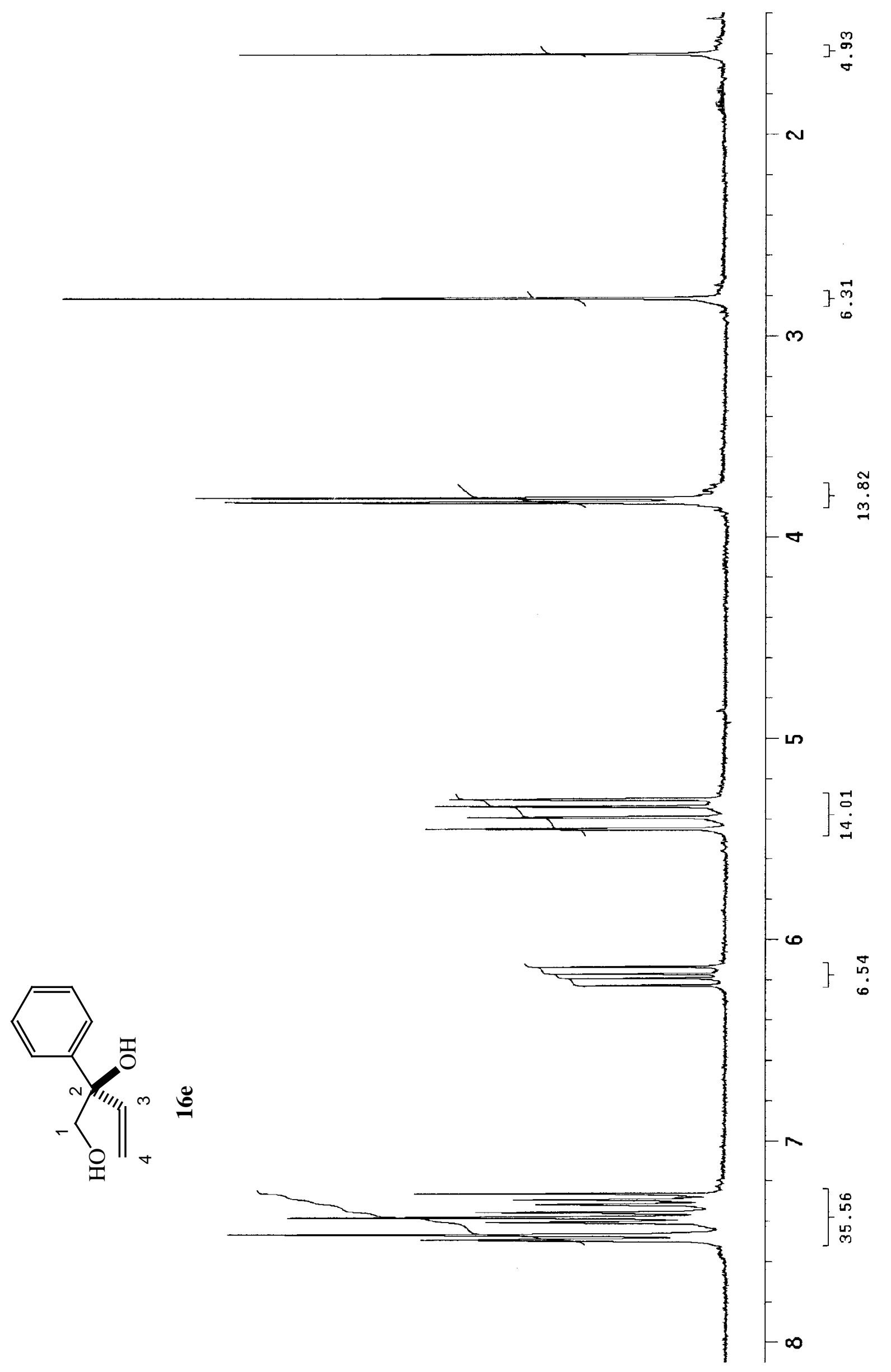

